\title{
Improved and robust spectral reflectance estimation
}

\author{
Jingjing Zhang ${ }^{\mathrm{a}, \mathrm{b}}$, Youri Meuret ${ }^{\mathrm{c}}$, Xiangguo Wang ${ }^{\mathrm{a}, \mathrm{b}}$, and Kevin A.G. Smet ${ }^{\mathrm{c} *}$ \\ ${ }^{a}$ School of Automation, China University of Geosciences, Wuhan, China; ${ }^{b} H u b e i$ Key \\ Laboratory of Advanced Control and Intelligent Automation for Complex Systems, Wuhan, \\ China; ${ }^{c}$ ESAT/Light\&Lighting Laboratory, KU Leuven, Ghent, Belgium \\ *kevin.smet@kuleuven.be
}

\begin{abstract}
Accurately estimating spectral reflectance functions from color camera images is a hot research subject that demonstrates tremendous potential for illuminating engineering and computer vision applications. However, the impact of the illumination spectrum and camera responsivity (system functions) to the estimation accuracy has not been systematically studied so far, nor the impact of 'training' spectral reflectance set. In this study, a dual imaging reflectance optimization system is used based on a neural network and optimal system functions that are resp. trained and optimized using several sample sets. Simulations showed that such optimal systems, trained and optimized with the IES TM30 spectral reflectance set, can have a substantially higher estimation accuracy compared to 'real' systems composed of commercially available projector spectra and camera responsivities and that they are sufficiently robust under small changes in system function peak wavelength and spectral width due to changes in working temperature or with passing time. An analysis of the impact of the specific sample set database adopted for neural net training on estimation accuracy showed that training with the IES set results in good and stable performance, even for other sample sets and different illumination spectra. Training with the spectrally uniform IES spectral reflectance set is therefore advised for general purpose, high-accuracy reflectance estimation systems. A comparison with a state-ofthe-art method shows that the proposed method has a higher color prediction accuracy and a significantly shorter running time for realistic images with high resolution.
\end{abstract}

Keywords: reflectance estimation; color accuracy; IES TM30 sample set; spectral optimization; dual imaging system 


\section{Introduction}

The spectral reflectance of a surface is a unique property that is independent of the impinging illumination (Healey G 1991; Dana 2016), and is responsible, along with illumination's spectral power distribution (SPD), for the surface's (object's) color appearance. The spectral reflectance information is therefore useful for illuminating engineering applications (Durmus et al. 2020), such as color tuning (Zhang J. et al. 2017; Durmus and Davis 2018), visual enhancement (Wang H et al. 2018; Shen et al. 2019), energy saving (Durmus and Davis 2015; Zhang JJ et al. 2019), and computer vision application, such as surface/material recognition and characterization (Tominaga Shoji and Okajima 2000; Tu et al. 2015), for image enhancement (Fu X et al. 2015), for color constancy (Dixon and Shapiro 2017) and for geometry (shape) estimation from shading (Oxholm and Nishino 2016). Moreover, it is also useful in realistic material reproduction under a variety of illumination conditions in computer graphics (Filip et al. 2017) and in relighting (Xing et al. 2010), where multispectral reflectance approaches (Shrestha et al. 2011; Khan et al. 2013) can hardly meet the requirements. Although surface hyperspectral reflectances can be simultaneously obtained

for an entire scene by a hyperspectral camera, or can be obtained by active basis light illumination (Lam et al. 2013), these methods remain costly (Nguyen et al. 2014) and is one of the reasons that reflectance information remains limited in commercial computer vision and signal processing.

Hyperspectral reflectance estimation from color images obtained using regular cameras is therefore a hot research topic (Deeb et al. 2019). Several techniques have been adopted. The simplest methods use a single camera, resulting in only three signals (RGB) from which to estimate the spectral reflectance (Maloney and Wandell 1986). For improved 
accuracy, additional signals have been generated by placing carefully selected filters in front of a light source (Chi et al. 2010) or in front of a monochrome camera (Tominaga S. 1996). The downside is that switching filters is time-consuming. Other systems, offering better timeresolution and ease of control, used LED clusters (Park et al. 2007) or a DLP projector (Han et al. 2013) in combination with an RGB camera. Nevertheless, compared with hyperspectral cameras, the estimation accuracy can hardly satisfy requirements for many practical applications. Therefore, in recent years, lots of research has been devoted to improving estimation accuracy by making the most of whatever signals are available using techniques such as Principle Component Analysis (PCA) (Zhang X and Xu 2008; Shrestha et al. 2011; Liu Z et al. 2013; Oh et al. 2016; Xiao et al. 2016; Liu Z et al. 2017), neural networks (Nguyen et al. 2014), regression approaches (Heikkinen Ville et al. 2016; Heikkinen V. 2018), Wiener estimation (Urban et al. 2009), deep learning (Georgoulis et al. 2017), and colorimetric methods (Cao et al. 2017; Nahavandi and Tehran 2017; Zhang X et al. 2017).

Although, reflectance estimation accuracy can be improved by adopting various estimation methods (Heikkinen Ville et al. 2008), existing research on the impact of light source spectrum and camera responsivity is limited. The effect of the camera sensitivity onto the spectral reconstruction accuracy are evaluated and proved significant in (Arad and BenShahar 2016), then methods for improving reflectance estimation accuracy by finding or designing optimal camera sensitivities (Arad and Ben-Shahar 2016; Fu Y et al. 2018; Nie et al. 2018) are proposed as well. Note that both light sources and cameras are essential in the imaging and estimation process. Therefore, for improving spectral reflectance estimation accuracy, separately optimizing the light source spectra and the camera sensitivities can miss important interactions between the two. Currently, there is no systematic study of their 
impact, nor is it known which combination of illumination spectrum and camera responsivity is most appropriate for accurate spectral reflectance estimation. In addition, the spectral reflectance set used in the training or estimation process can have a substantial impact. It is also unclear if a single training set (and if so, which one?) can be used for general purpose applications, and how robust the estimation is for spectral reflectances from a different set (e.g. containing different pigments and hence spectral characteristics), nor how robust the estimation is under different illumination conditions.

In this work, spectral reflectances are estimated, using a neural network, from the signals obtained from a dual imaging system composed of a three channel light source (e.g. a digital light processing (DLP) projector) and a digital RGB color camera, similar to (Han et al. 2013). To more accurately simulate a real imaging environment, photon noises are included. In a first stage, the dual imaging system is simulated using real illumination spectra and camera responsivities - henceforth jointly referred to as 'system spectral functions' from commercially available hardware. In a second stage, optimized system spectral functions are derived by minimizing the root-mean-square-differences (RMSD) between actual and estimated spectral reflectances and their mean color difference when illuminated by an equal-energy white (CIE illuminant E). The reflectance estimation performance of the real system and the simulated (optimized) system are compared and analyzed. In a third stage, the robustness of the optimized system spectral functions to small variations is analyzed. Then, the impact of the spectral reflectance set used during training and optimization is systematically investigated. The paper ends with a comparison of the estimation accuracy, illuminant metamerism robustness and the running speed of the proposed method with those of Liang's method (Liang et al. 2019), one of the latest methods proposed in literature. 


\section{Spectral reflectance estimation}

\subsection{Basic image formation}

When a surface characterized by a spectral reflectance, $R(\lambda)$, and illuminated by a light source with spectral power distribution (SPD), $S(\lambda)$, is imaged by a camera pixel with spectral responsivity, $C(\lambda)$, then the pixel output can mathematically represented by (Park et al. 2007):

$$
O=\int_{400 \mathrm{~nm}}^{700 \mathrm{~nm}} S(\lambda) \cdot R(\lambda) \cdot C(\lambda) d \lambda
$$

where, $\lambda$ is the wavelength, ranging from $400 \mathrm{~nm}$ to $700 \mathrm{~nm}$ in camera-sensitive wavelength range.

For color cameras, there are typically three spectral responsivity curves, commonly designated as the $\mathrm{R}(\mathrm{ed}) \mathrm{G}(\mathrm{reen}) \mathrm{B}(\mathrm{lue})$ camera channels. A single pixel would therefore provide three signals from which the spectral reflectance is to be recovered.

\subsection{Photon noise}

In real imaging systems, photon noise, shot noise, read noise, and ADC noise contribute in varying proportions at different signal levels, leading to an overall signal noise which is dependent on scene brightness (Hasinoff 2014). While read noise and ADC noise can be accounted for by signal independent Gaussian additive noise; photon noise, whose variation is linearly proportional to the signal, is the most prominent in a system with high brightness (Prucnal and Saleh 1981; Healey GE and Kondepudy 1994; Jiang and Gu 2012). In practice, photon noise is often modeled using a Poisson distribution or a Gaussian distribution with variance equal to the mean (Foi et al. 2008; Liu C et al. 2008). In this study, photon noise has been added to the pixel output signals to simulate real world camera operation. 


\subsection{Dual Imaging System for Increased Accuracy}

To increase the available information (signals) and hence accuracy, in this work a dual imaging system is used which is composed of a 3-channel light source (e.g. a data projector) and a 3-channel color camera (Han et al. 2013). By sequentially imaging a surface under each of the three light source channels, nine signals (monochromatic images) are available for reflectance estimation. However, the specifics of the lighting SPDs and camera responsivity curves can have an important impact on achievable accuracy, as the nine channel outputs are not necessarily uncorrelated, leading to information redundancy.

Two types of dual systems will be explored in this work: real systems composed of commercially available projector (lighting) SPDs and camera responsivities, and theoretical dual systems with simulated lighting SPDs and camera responsivity curves. Note that although much of what follows is valid for any type of RGB lighting, projectors were specifically chosen for their capability of providing spatially tunable lighting important in certain practical applications of the system (Zhang J et al. 2019).

\subsubsection{Real systems}

Real system projectors are typically light-emitting-diode (LED) or xenon-lamp based (Technologies ; Packer et al. 2001; Delica and Blanca 2007; Samson and Blanca 2007; Familiarization with Properties of Light Sources 2010; Trussell 2011; Stirman et al. 2012; Han et al. 2013; Ali et al. 2014; Perlova 2014; AEON Imaging 2015; Grusche and Theilmann 2015). Cameras include professional DSLRs, point- and-shoot, industrial and mobile cameras, and are manufactured by different corporations, i.e. Canon, Nikon, Sony, Point Gray (Jiang et al. 2013; Coburn et al. 2018; Burggraaff et al. 2019; de Miguel et al. 2019). Note that in order to guarantee accurate colorimetric reproduction for various surfaces 
illuminated by various illumination spectra, i.e. to reduce effects of metamerism, a set of camera responsivities is required to obey the Luther condition (Schubert and Kim 2005). The latter requires the responsivities to be a linear transformation of the color matching functions (Ohno Yoshi 2005). The potential for these color errors is typically assessed by the digital still camera sensitivity metamerism index (DSC/SMI). This index has a maximal value of 100 and uses the calculation framework of the general color rendering index $R \mathrm{a}$ (Xie B. et al. 2016). For a fair comparison with our optimized camera responsivities, only the nine sets of camera responsivities with SMI values higher than 95 from a total of 35 collected cameras responsivities (Jiang et al. 2013; Coburn et al. 2018; Burggraaff et al. 2019; de Miguel et al. 2019) are further used in this study. Therefore, a total of 13 projector SPDs and 9 camera responsivities have been collected and all possible combinations (117) have been made. The set of commercially available system has been plotted in Fig. 1.
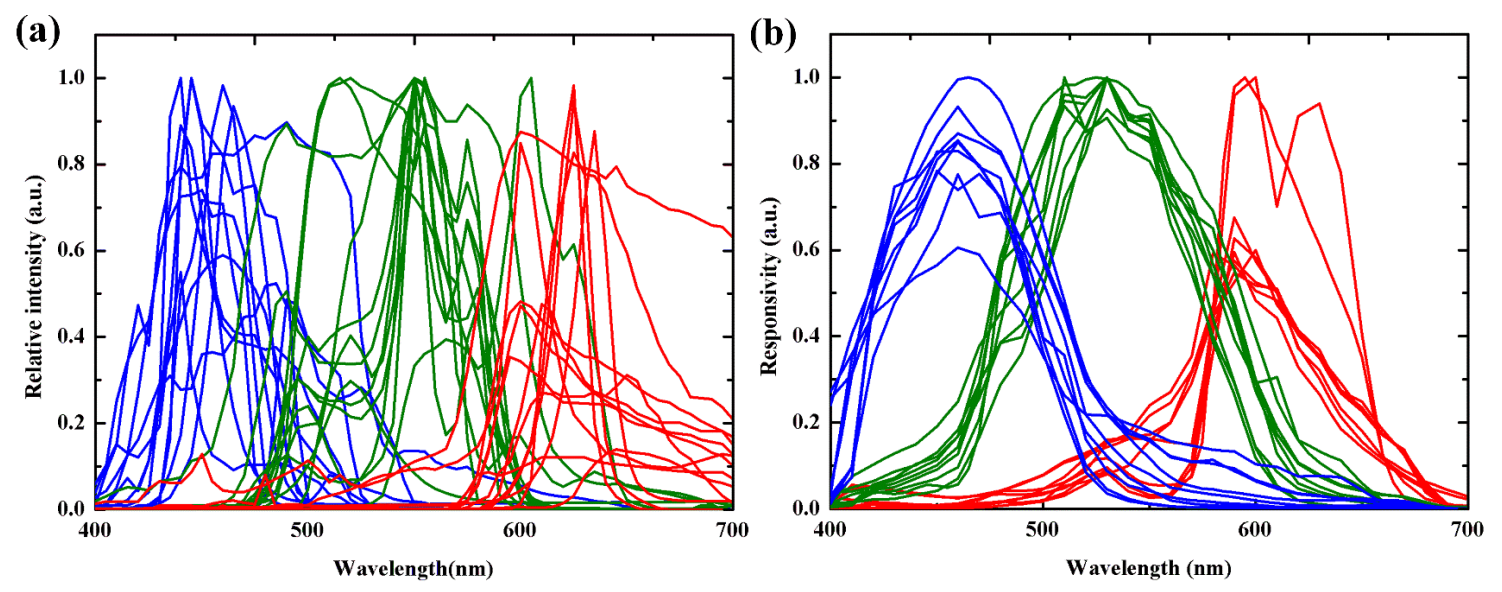

Fig. 1 . The set of commercially available system functions (a. 13 projector SPDs; b. 9 camera responsivities).

\subsubsection{Theoretical systems}

The theoretical systems were simulated with the aim of finding one or more optimal system 
function sets that minimize the deviation between known spectral reflectance functions (ground-truths) and their estimates (see next section). Due to the merits of wide gamut area, long lifetime, high contrast ratio, and high luminous efficacy (Schubert and Kim 2005), monochromatic light-emitting diodes (LEDs) have gradually replaced the traditional projector light sources. Monochromatic LEDs were therefore adopted as projector light sources and their SPDs were approximated by Ohno's modified Gaussian model (Ohno Yoshi 2005). Peak wavelengths and Full-Width-at-Half-Maximum (FWHM) varied respectively from $400 \mathrm{~nm}$ to $700 \mathrm{~nm}$ and from $20 \mathrm{~nm}$ to $300 \mathrm{~nm}$ (Ohno Yoshi 2005; Xie B. et al. 2016). In practice, optimal projector spectra can be fabricated by a combination of monochromatic LEDs, quantum dots, and phosphors (Xie Bin et al. 2017; Xu et al. 2017).

Modeling camera responsivity curves is also a topic worthy of research. Radial basis function models (Zhao H et al. 2009) and PCA-based models (Jiang et al. 2013) have been proposed for camera responsivity curve modeling. However, the above models have limited flexibility compared to parametric functions, such as Gaussians or rounded triangles. The latter were found to be able to approximate real world camera responsivities quite accurately, as can be seen from Fig. 2. Rounded triangle functions, as also used in (Smet K. A. G. et al. 2013) to model a set of spectrally uniform spectral reflectance functions for use in color rendering fidelity index (CRI2012), were therefore adopted to simulate camera responsivities. Peak wavelengths varied from $400 \mathrm{~nm}$ to $700 \mathrm{~nm}$, forward slope width and rear slope width respectively varied from 10 to $150 \mathrm{~nm}$. In practice, optimal camera responsivities can be obtained by color filters (Ng and Allebach 2006; Li S-X 2018). 

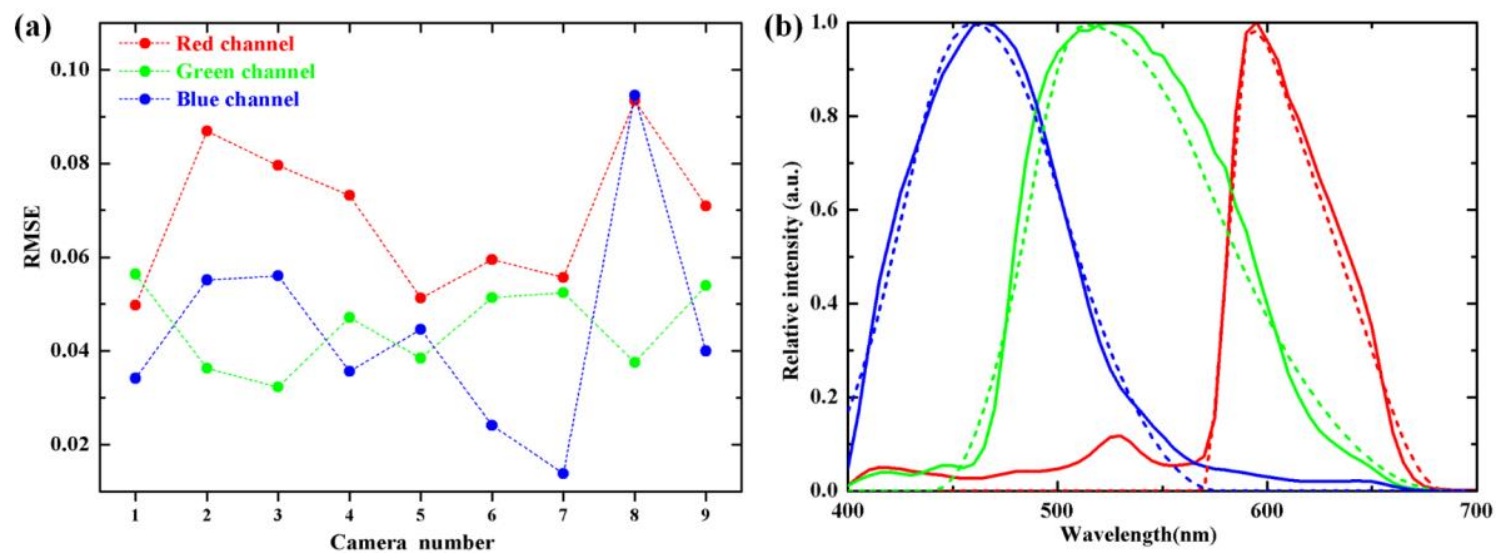

Fig. 2. (a) RMSE goodness-of-fit between nine real camera responsivity curves and nine rounded triangle approximations. (b) Example of a set of real (solid lines) and simulated (dashed lines) camera responses.

\subsection{Spectral reflectance estimation using neural networks}

The spectral reflectance of a surface is estimated from the nine signals using a neural network approach for a convenient mapping between light source spectra, spectral reflectances and camera-specific RGB values rather than other methods (Nguyen et al. 2014). In this work a 3-layer radial basis neural network (using the newrb function in Matlab) is adopted. The input layer is a 9-element vector consisting of the camera output RGB values 'captured' under each of the 3 light source (projector) spectra. The hidden layer has neurons with a Gaussian radial basis function. The output layer has neurons with a linear function, which adds the weighted output of the hidden layer. The latter takes as input the weighted Euclidean distance between the input vectors. Initially the hidden layer has no nods. The following steps are repeated until the nods of the hidden layer increases to 200 :

1) The network is simulated.

2) The input vector with the greatest error is found.

3) A neuron with radial basis function is added with weights related to that vector.

4) Weights of the output layer are redesigned to minimize error. 
The neural network is trained using a large number of known spectral reflectance functions (ground-truth) and their simulated signals (nine for each surface). The training set was composed of 2928 samples $(60 \%)$ randomly drawn from the 4880 samples present in the extended reference spectral reflectance set of IES TM-30 (David et al. 2015; IES 2018). These samples are uniformly distributed in both color space (CAM02-UCS (Ng and Allebach 2006)) and wavelength space (Li C et al. 2012; Smet Kevin A. G. et al. 2016). As mentioned in the previous section, the system spectral functions were either commercially available or were simulated using an Ohno's LED model (light source SPDs) or using rounded triangles (camera responsivities).

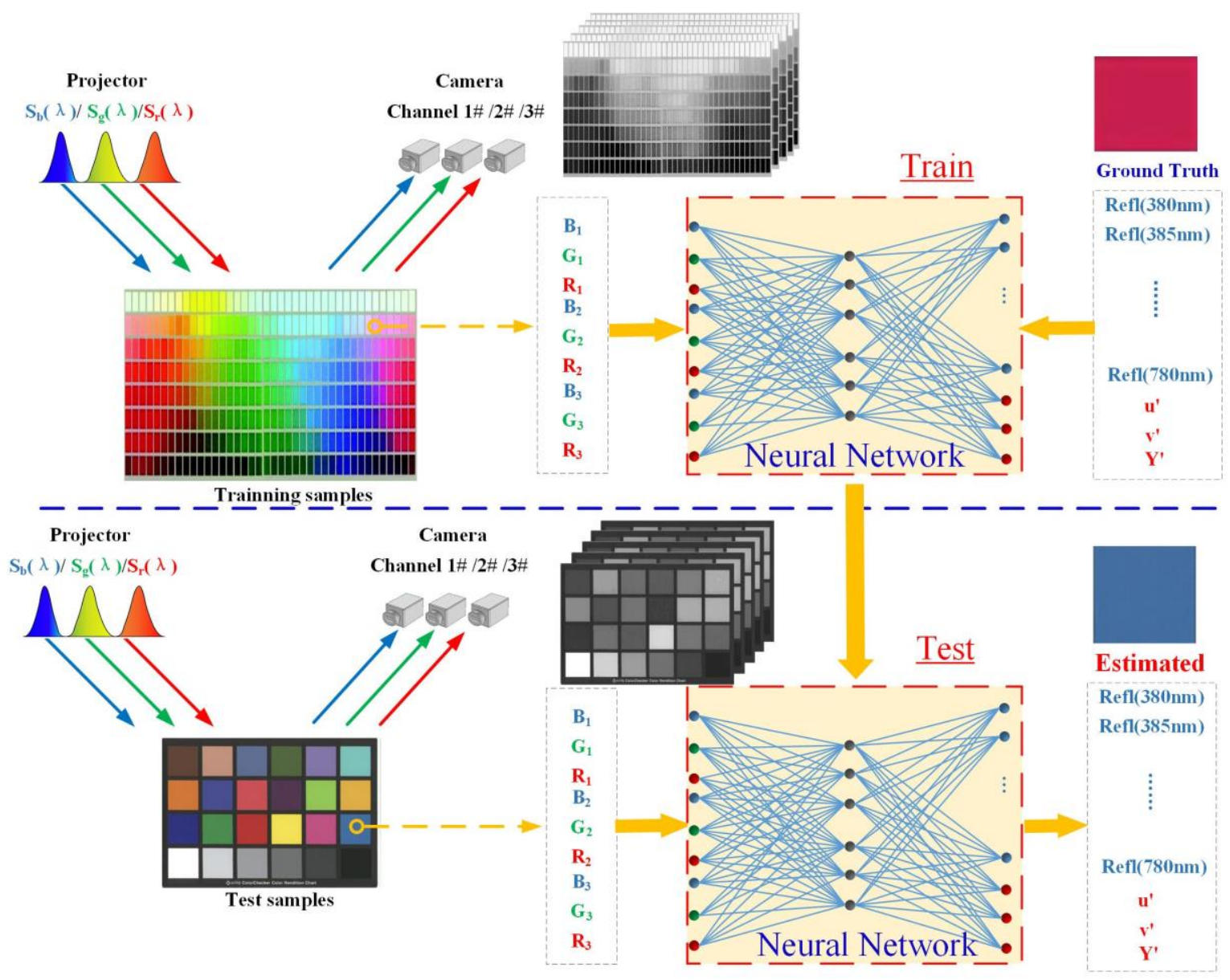

Fig. 3. Neural network based reflectance estimation schematic diagram. 
Final spectral reflectance estimation accuracy of the trained/optimized system was evaluated using four fitness measures: the mean Root-Mean-Square-Error (RMSE) and Goodness-of-Fit-Coefficient (GFC) of the estimated spectral reflectance and the ground truth for spectral accuracy assessment (l'Eclairage 2005; Cao et al. 2017); and the mean CIE 1976 $u^{\prime} v^{\prime}$ chromaticity differences $\left(\Delta u^{\prime} v^{\prime}\right)$ and luminance differences $(\Delta Y)$ between the estimated and ground-truth chromaticity and luminance values obtained under some reference illuminant for color accuracy evaluation (Urban et al. 2009; Zhang X et al. 2017). Good color accuracy is crucial for various human vision and colorimetry applications. The four parameters are given by:

$$
\left\{\begin{array}{l}
R M S E=\sqrt{\frac{1}{N} \sum_{i=1}^{N}\left(R_{i}-\hat{R}_{i}\right)^{2}} \\
G F C=\frac{1}{N} \sum_{i=1}^{N}\left|R_{i} \hat{R}_{i}\right| /\left(\sqrt{\sum_{i=1}^{N} R_{i}^{2}} \sqrt{\sum_{i=1}^{N} \hat{R}_{i}^{2}}\right) . \\
\Delta u^{\prime} v^{\prime}=\sqrt{\left(u^{\prime}-\hat{u}^{\prime}\right)^{2}+\left(v^{\prime}-\hat{v}^{\prime}\right)^{2}} \\
\Delta Y=|Y-\hat{Y}| / Y
\end{array}\right.
$$

where, $R_{i}$ and $\hat{R}_{i}$ refer to the ground truth and the estimated value of the reflectance, respectively; $u^{\prime}$ and $v^{\prime}$ are the ground truth in the CIE1976 chromaticity diagram, $\hat{u}^{\prime}$ and $\hat{v}^{\prime}$ are the corresponding estimated values; $\Delta u^{\prime} v^{\prime}$ is the $u^{\prime} v^{\prime}$ deviation in the CIE1976 chromaticity diagram; $Y$ and $\hat{Y}$ respectively designate the ground truth and the estimated value calculated using the CIE 1931 color matching functions (cfr. 1924 V-lambda curve); and $\Delta Y$ designates the deviations between the ground truth and the estimated value of $Y$. In this work the equi-energy-white (CIE illuminant E) has been adopted as reference illuminant. $N$ is the sampling number of spectral reflectance in visible spectrum, for spectral wavelength ranges from 400 to $700 \mathrm{~nm}$ at $5 \mathrm{~nm}$ intervals, $N=61$. 
After training, the neural net estimator was tested using a set of test spectral reflectance functions composed of $20 \%$ of the IES TM-30 sample set (randomly drawn from the remaining $40 \%$ ). The process of training and testing a neural network for an arbitrary set of system functions is illustrated in Fig. 3 .

In the case of the derivation of the optimal set of simulated system functions, the above process (training followed by testing) was repeated many times during each step of an optimization process that varied the peak wavelengths and spectral widths of the simulated light source SPDs and camera responsivity curves until their optimal values were obtained. Optimizing the 2-by-6 parameter matrix (6 parameters for 3 projector SPDs and 3 camera responsivities) is a complex nonlinear programming (NLP) problem. A genetic algorithm (GA) has been selected, as it has been shown to have faster global convergence and higher computational efficiency for complex NLP problems than other optimization methods, such as the ant colony and particle swarm algorithms (Deb 2000), as well as high reflectance estimation accuracy (Zhang J. et al. 2017).

The GA algorithm ran for 50 generations with a population size of 40 , a generation gap of 0.9 , chromosome accuracy of 25 , and chromosome pair cross-over probability of 0.7 . The flowchart of the optimization process is shown in Fig. 4. The first step in the GA optimization process (see flowchart in Fig. 4) is the random initialization of the parameter chromosomes (peak wavelengths and the FWHMs of projector SPDs and camera responsivities) of each member of the population. Each chromosome thus represents a set of system functions. The next step is to train a neural network for each of these sets. During testing, the performance of the trained nets is then evaluated using a fitness value $f$, which is a weighted mixture of the mean RMSE values, the mean $\Delta u^{\prime} v^{\prime}$ values, and the mean $\Delta Y$ values between the ground-truth and estimated spectral reflectances generated by each neural net. 
Since color difference is a key parameter in practical color science applications (Vora and Trussell 1993; Sharma and Trussell 1997), different combinations of the loss functions have been tested and it was found that the weights as shown in Eq. 3, result in a good tradeoff between RMSE values, $\Delta u^{\prime} v^{\prime}$ values (indicating good and stable colorimetric accuracy) and $\Delta Y$ values:

$$
\left\{\begin{array}{l}
\text { Minimum: } f=\sqrt{R M S E^{2}+10 \Delta u^{\prime} v^{\prime 2}+(\Delta Y / 100)^{2}} \\
\text { Constraint: } S M I \geq 95
\end{array} .\right.
$$

The fitness function $F$ of the GA is defined as the sum of the fitness function $f$ and the penalty terms depend on the constraint, which was also used in our previous work (Zhang J. et al. 2017; Zhang JJ et al. 2017).

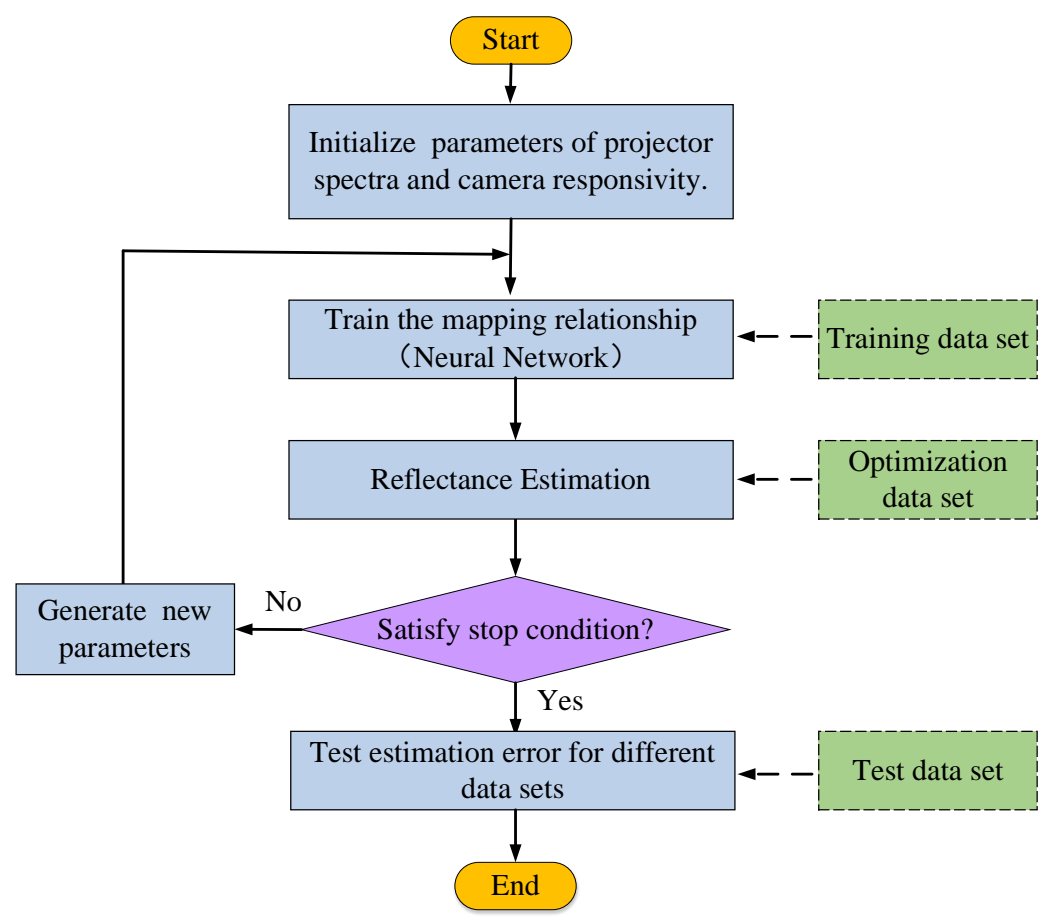

Fig. 4. Flowchart of the optimization process.

When the stopping criterium (50 genetic iterations) is not reached, a new population (next generation) of chromosomes is generated by crossing, selecting and mutating the chromosomes of the current population and the process starts over. In case the stopping 
criterium is reached, the optimal system (optimized neural network and set of system functions) is evaluated using the remaining $20 \%$ (i.e. 976) of samples from the IES TM-30 set using all 4 goodness-of-fit measures (Eq. 3).

For both real systems and theoretical systems, the robustness of the (optimal) trained neural network for spectral reflectance estimation of sets other than the IES TM-30 set (used during training) has been checked using the full Leeds 100000 reflectance data base (Smet K. A. G. et al. 2013), the full set of 1269 Munsell samples (Jiang and Gu 2012) and the 24 Macbeth ColorChecker samples (Co 1997).

\subsection{Robustness of optimal system functions under small parameter shifts}

As the working temperature increases or as time goes by, the peak wavelengths and FWHMs of LEDs and camera responsivities shift (Zhang JJ et al. 2013; Raypah et al. 2017). The robustness of reflectance estimation under small variations of the optimal projector spectra and camera responsivities, important for stable reflectance estimation accuracy in practical applications, was therefore investigated.

Robustness has been tested by systematically and individually shifting, after neural network training, each of the 12 system function parameters over a $\pm 4 \mathrm{~nm}$ range in steps of $1 \mathrm{~nm}$ and calculating the mean RMSE values for the Macbeth data set using the shifted system functions. Note that $4 \mathrm{~nm}$ wavelength shifts correspond to about $70 \mathrm{~K}$ and $80 \mathrm{~K}$

temperature shifts in the blue and red wavelength region respectively (Zhang JJ et al. 2013; Zhao Y et al. 2017). Finally, to test compound effects, a similar analysis has been performed by simultaneously shifting two system function parameters. 


\subsection{Impact on optimal system performance of the sample set database used for neural network training}

The impact of the specific sample set database adopted for training the neural net and optimizing the system functions has been investigated by performing system optimizations using three different training sample sets (the IES set (Li C et al. 2012; IES 2018), the Munsell set (Jiang and Gu 2012) and the Leeds 100000 set (Co 1997)) and analyzing the system performance in terms of mean RMSE between ground-truth and estimate spectral reflectances. To focus specifically on effects of possible differences in specific spectral characteristics of the samples in each set, and not potential differences generated by differences in sample size and sample gamut, three subsets of equal size and equal CAM02UCS color gamut are drawn from each of the 3 sample databases.

The flowchart of the selection process is shown in Fig. 5(a). First, the CAM02UCS color space is divided in equal sized cubes and from all samples present within a (non-empty) cube one sample is randomly selected. This process is repeated three times for each sample database to obtain a total of 9 sample sets ( 3 random subsets for each of 3 main reflectance sets). The next step is to determine the common gamut spanned by these 9 subsets. The common gamut size is characterized by the minimum CAM02UCS chroma (denoted as $C_{\min }$ ) of all 9 sets. All 9 subsets are then limited to have a gamut not larger than $C_{\min }$ by deleting all sampling cubes (and the samples they contain) which have a chroma larger than $C_{\min }$. This ensures that no set would have more extreme (higher chroma) samples than any of the others, which could otherwise bias the analysis results as high chroma samples are typically characterized by higher spectral variation. Next, the number of samples, i.e. the remaining non-empty cubes (denoted by $N_{\min }$ ), are counted and each set is further reduced to have a size equal to the smallest set by randomly selecting non-empty cubes and deleting their samples. 
This ensures that each set contains the same amount of samples. This process is repeated for cubes of varying size in order to end up with subsets which have a sufficiently large gamut and amount of remaining samples within this common gamut for neural network training and testing. Note that all CAM02UCS coordinates are calculated in a wavelength range of 380 $\mathrm{nm}$ to $780 \mathrm{~nm}$ for accurate CAM02-UCS coordinates in this section.

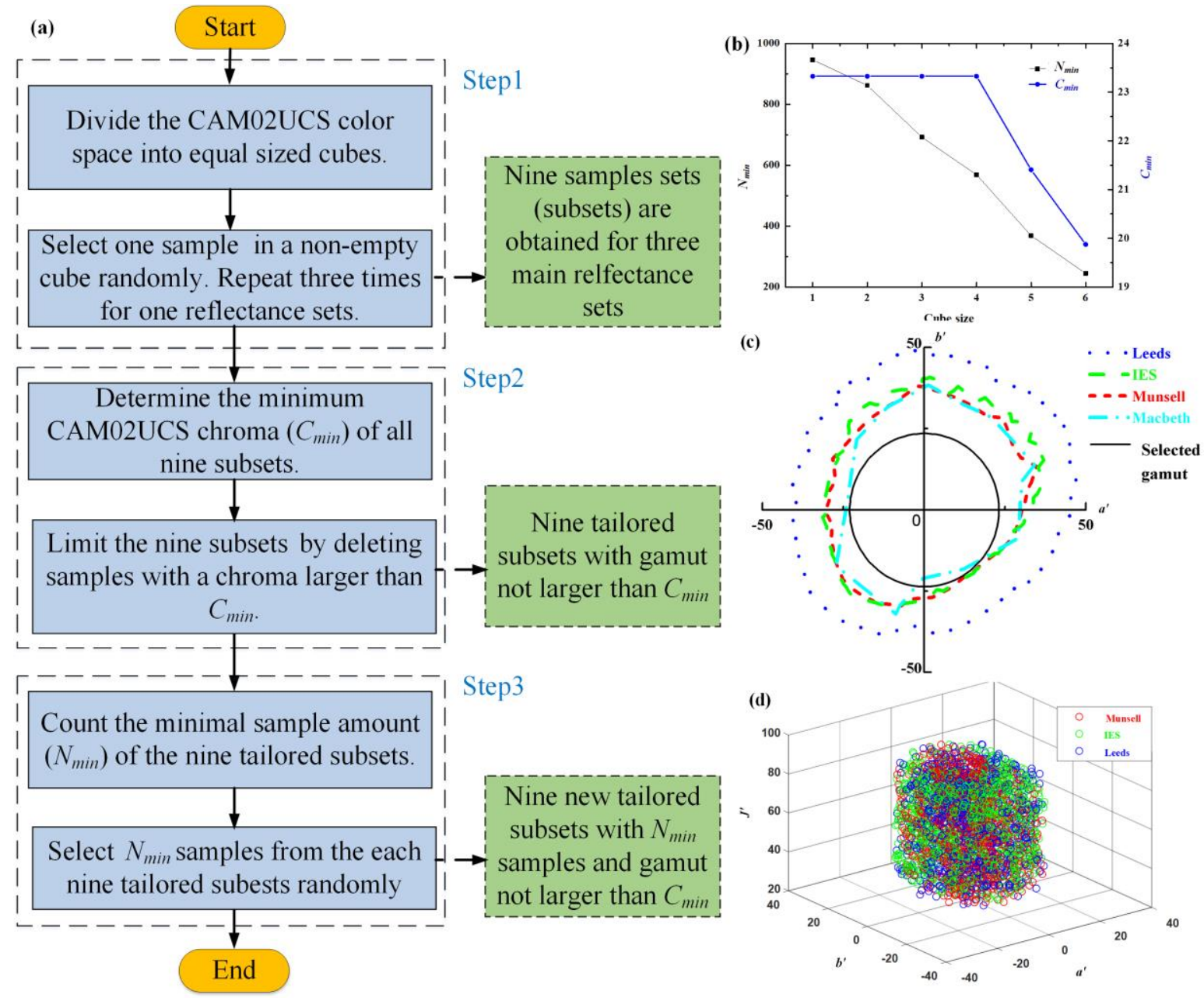

Fig. 5. (a) Flowchart of the selection process; (b) Sample set size, Nmin, and minimum chroma, Cmin, as a function of cube size; (c) Color gamut projections on the $a^{\prime} b^{\prime}$ plane for the Leeds (blue dotted line), IES (green long dashed line), Munsell (red short dashed line), Macbeth (cyan dash-dotted line) sample sets, and the common cylindrical volume (solid black line); (d) $J^{\prime} a^{\prime} b^{\prime}$ color coordinates of the selected samples for the 3 sample databases (Munsell: red circles, IES: green circles and Leeds: blue circles). 
The sample set gamut and sample set size vary with cube size as shown in Fig. 5(b). It can be seen that the $C_{\min }$ remain unchanged with the cube size increases from 1 to 3 , and then decreases with the cube size increases from 3 to 6 . In addition, the $N_{\min }$ decreases with the increasing cube size. For very small cube sizes (1-2), both $C_{\min }$ and $N_{\min }$ reach high values. (For a cube size of 1, $C_{\min }$ and $N_{\min }$ have values of 23.2 and 946, respectively). Color gamut projections on the $a^{\prime} b^{\prime}$ plane for the Leeds, IES, Munsell, Macbeth sample sets, and the common cylindrical volume are plotted in Fig. 5(c). A plot of $J^{\prime} a^{\prime} b^{\prime}$ coordinates of the final selected samples for each of the 3 sample databases, in the case of cube size 1, is shown in Fig. 5(d).

Using these 9 subsets optimal simulated system functions and associated neural networks have been derived for nine different training sets, each randomly drawn from and with a size of $60 \%$ of these subsets. As before, in each case, the fitness value, $F$ (see Eq. 3 ), describing the goodness-of-fit between ground-truth and estimated spectral reflectances, was minimized.

Performance was tested using the full Munsell set, full Macbeth data set, full IES data set, full the Leeds data set and $20 \%$ of the selected set for training, as well as these sets limited to the minimal cylindrical volume described above.

\subsection{A comparison of accuracy and running speed of the proposed method with Liang's method}

For comparison, the accuracy, color robustness to illuminant metamerism and running speed of the proposed method has been compared with one of the latest methods published in literature, the method of Liang (Liang et al. 2019). The latter estimates the spectral reflectance of a test sample as a weighted linear combination of the spectral reflectances of 
the samples in a training set (e.g. the CCSG samples). The training samples used in the estimation of each test sample are selected based on the (adaptively) weighted CIELAB Euclidean distance between the test sample and the training samples. For a fair comparison with the proposed method, Liang's method has also been extended (improved upon) to include photon noise and simulated optimized system functions. Photon noise was added as before, while the parameter chromosomes for system function optimization also included the spectral intensities of the three projector color components (cfr. weighted sum), in addition to the peak wavelengths and the FWHMs of the projector SPDs and camera responsivities. In each calculation, the parameter $k$ (determining the effective range of training samples) was selected, from a range of 1 to 10, that resulted in the minimal RMSE value.

Both the original and the extended methods of Liang, with and without photon noise, were compared with the proposed neural network approach. Training and optimization of Liang's methods and the proposed methods was done three times using three different training sets randomly drawn from and with a size of $60 \%$ of the selected IES subset, which was limited to the minimal cylindrical volume and described in Section 2.6. Accuracy was assessed as the mean RMSE between the ground-truth and the estimated spectral reflectances of the full IES, full Munsell and full Leeds sample sets (which were also limited to the minimal cylindrical volume) and as the corresponding $\Delta u^{\prime} v^{\prime}$ color differences under illuminant E (same as used during 'training'). In addition, to estimate accuracy (robustness) of the estimation methods under different or unknown illumination spectra, the $\Delta u^{\prime} v^{\prime}$ color differences between ground-truth and estimated (under illuminant E) spectral reflectances were obtained when illuminated by the 318 spectral power distributions listed in the TM3015 excel calculator (David et al. 2015). 
In addition to estimation accuracy, running speed is another key performance factor for practical applications, especially for those that involve spectral reflectance estimation in real scenes. Running speed depends on the hardware setup (in this study Intel Core(TM) CPU i9-9820X, 32GB RAM), the method's algorithmic implementation, the image resolution and the color diversity of the scene, i.e. the number of pixels with unique RGB values. Especially Liang's method is expected to be highly sensitive to the color diversity of the scene and the image resolution, as for each unique RGB triple, it requires color distance calculation for all training pixels. Efficient implementation of Liang's method is therefore crucial. Different implementation methods, such block diagonal matrix calculations and inverse matrix operation were tested. Results for the most efficient method are reported. Typical color diversity in indoor scenes has been estimated from a database containing a total 15620 images, grouped into 67 indoor categories (Quattoni and Torralba 2009). On average, an image contained $18.3 \%$ unique pixels. Running speed, after training (and system optimization) was determined as a function of the number of unique pixels in a scene, i.e. as the number of unique validation samples to be estimated.

\section{Results and discussion}

\subsection{Spectral reflectance estimation with real projector and camera functions}

Each of the 117 neural networks, corresponding to all possible combinations of the 13 commercially available projectors and 9 cameras, were trained using $60 \%$ of the IES set and then evaluated using $20 \%$ of the IES set as well as the full Leeds, the full Munsell and the

full 24 Macbeth sets. This was repeated 5 times, each time selecting new $60 \%$ training and $20 \%$ test IES subsets and adding random photon noise to the 9 signals, during both the 
training and evaluation of the neural network.
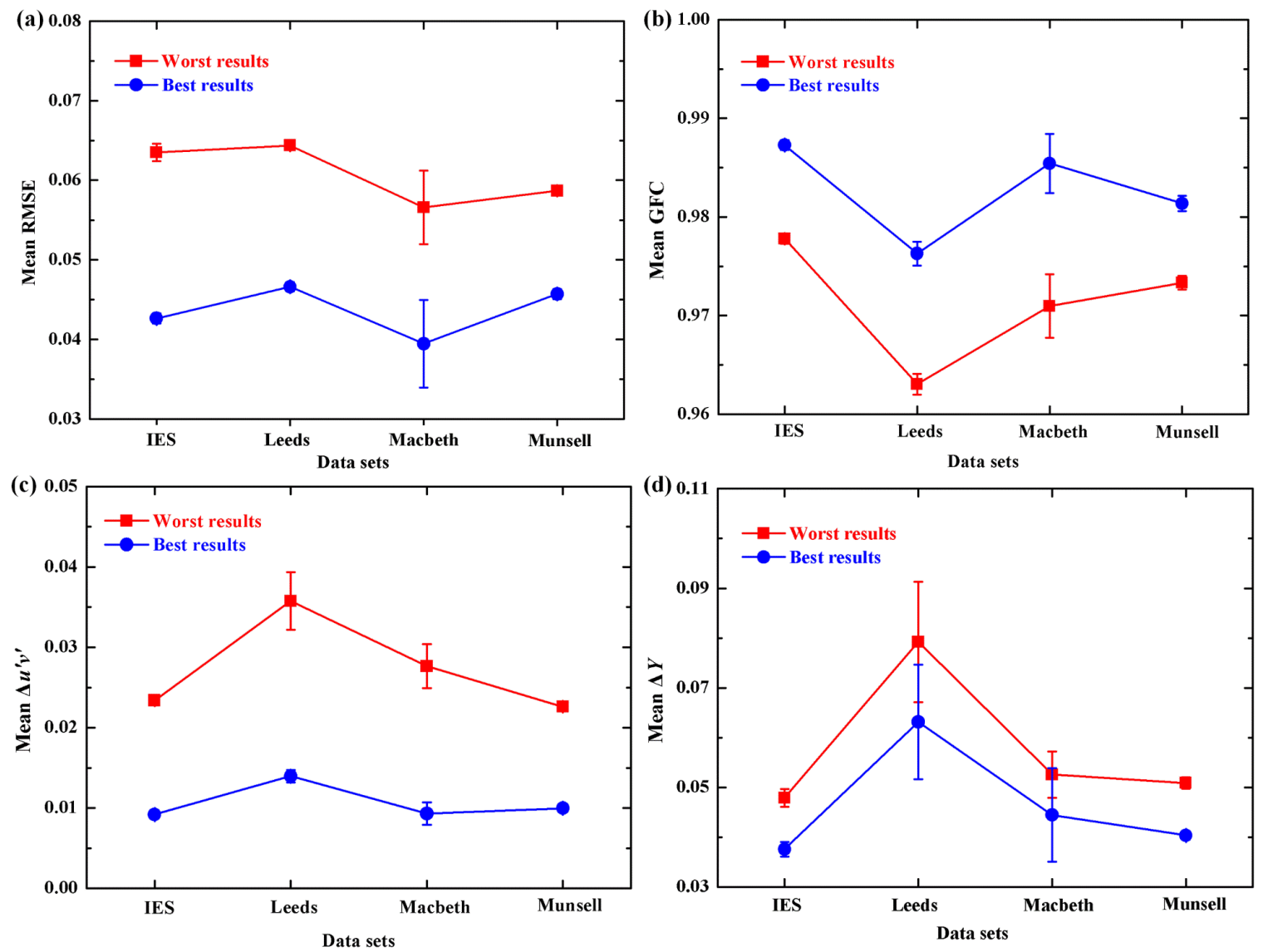

Fig. 6. Performance values of the best and worst system functions chosen using the RMSE of IES set with real projectors and cameras in terms of a) mean RMSE; b) mean GFC; c) mean $\Delta u^{\prime} v^{\prime}$; d) mean $\Delta Y$.

The mean results (over the 5 repeats) of the 4 evaluation measures for the best and worst system function, selected based on the mean RMSE values, are shown in Fig. 6. It can be seen from Fig. 6 that the "Mean RMSE" values of 9 cameras with SMI $\geqslant 95$ tend to vary from 0.043 to 0.063 for the IES set, 0.047 to 0.064 for the Leeds set, 0.039 to 0.057 for the Macbeth set, 0.046 to 0.059 for the Munsell set, indicating reflectance estimation accuracy differs greatly for different combinations of real projector spectra and camera responsivities. In addition, the performances of GFC, $\Delta u^{\prime} v^{\prime}$, and $\Delta Y$ also vary greatly for different 
combinations of real projectors and cameras. Consequently, light source spectra and camera responsivity play a very important role in the performance of reflectance estimation accuracy.

\subsection{Spectral reflectance estimation with optimal simulated projector and camera functions}

Optimal simulated system functions and associated neural networks have been derived for three different training sets, each randomly drawn from and with a size of $60 \%$ of the IES TM-30 data set. In each case, the fitness value, $F$, in Eq. 3 was minimized subject to an SMI $\geqslant 95$ constraint for the camera responsivities. The average optimal results of the mean RMSE values for different validation sample sets are shown in Fig. 7(a). Note the small deviation bars. For comparison the best results and the mean results obtained using real system functions are also shown. It can be found from Fig. 7(b) that the fitness function value decreases rapidly from 40.65 to 0.058 with increasing number of genetic algorithm generations. Compared with the best results of real projector and camera, the mean RMSE value of the IES set can be substantially decreased from 0.042 to 0.034 by optimizing the system functions (note that photon noise was included in both cases). Note also that the optimized system functions obtained for the IES set lead to substantially reduced mean RMSE values compared to the real system functions, which also show good performance for the Leeds, the Macbeth and the Munsell set. In addition, ignoring photon noise further reduces the mean RMSE values, as shown in Fig. 7(a). 

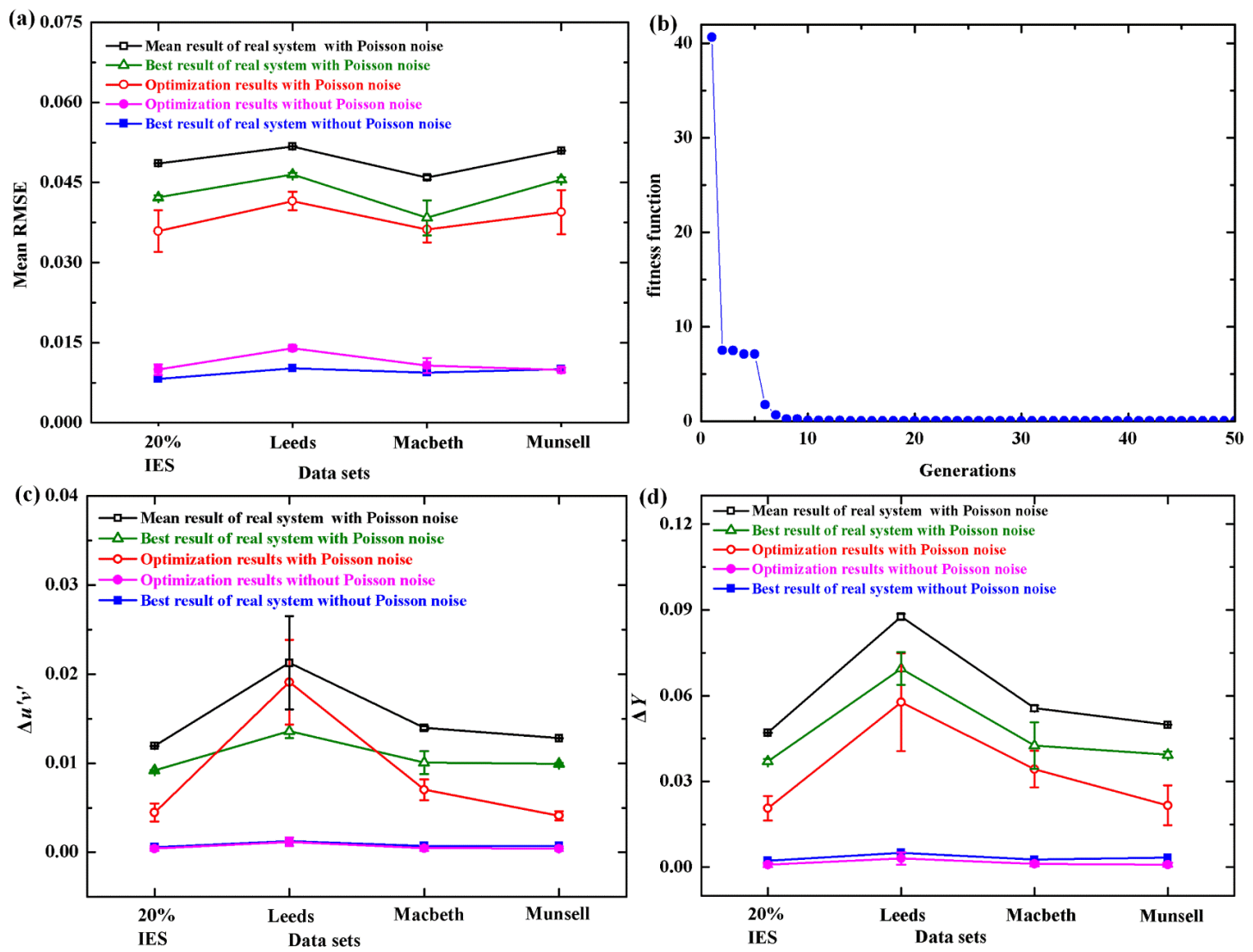

Fig. 7. RMSE values of optimized and real systems for several test spectral reflectance sets. (a. mean RMSE improvement after optimization for different data sets; b. fitness function as a function of increasing genetic algorithm generation; c. $\Delta u^{\prime} v^{\prime}$ improvement after optimization; d. mean $\Delta Y$ improvement after optimization).

As a visual illustration of the improved estimation accuracy of optimized versus real system functions, the estimated and ground-truth spectral reflectance curves have been plotted in Fig. 8 for a select number of samples of the Macbeth ColorChecker set. It is clear that the visual agreement for both types of system is quite good, but that the real system functions tend to lead larger deviations, especially at longer wavelengths. 


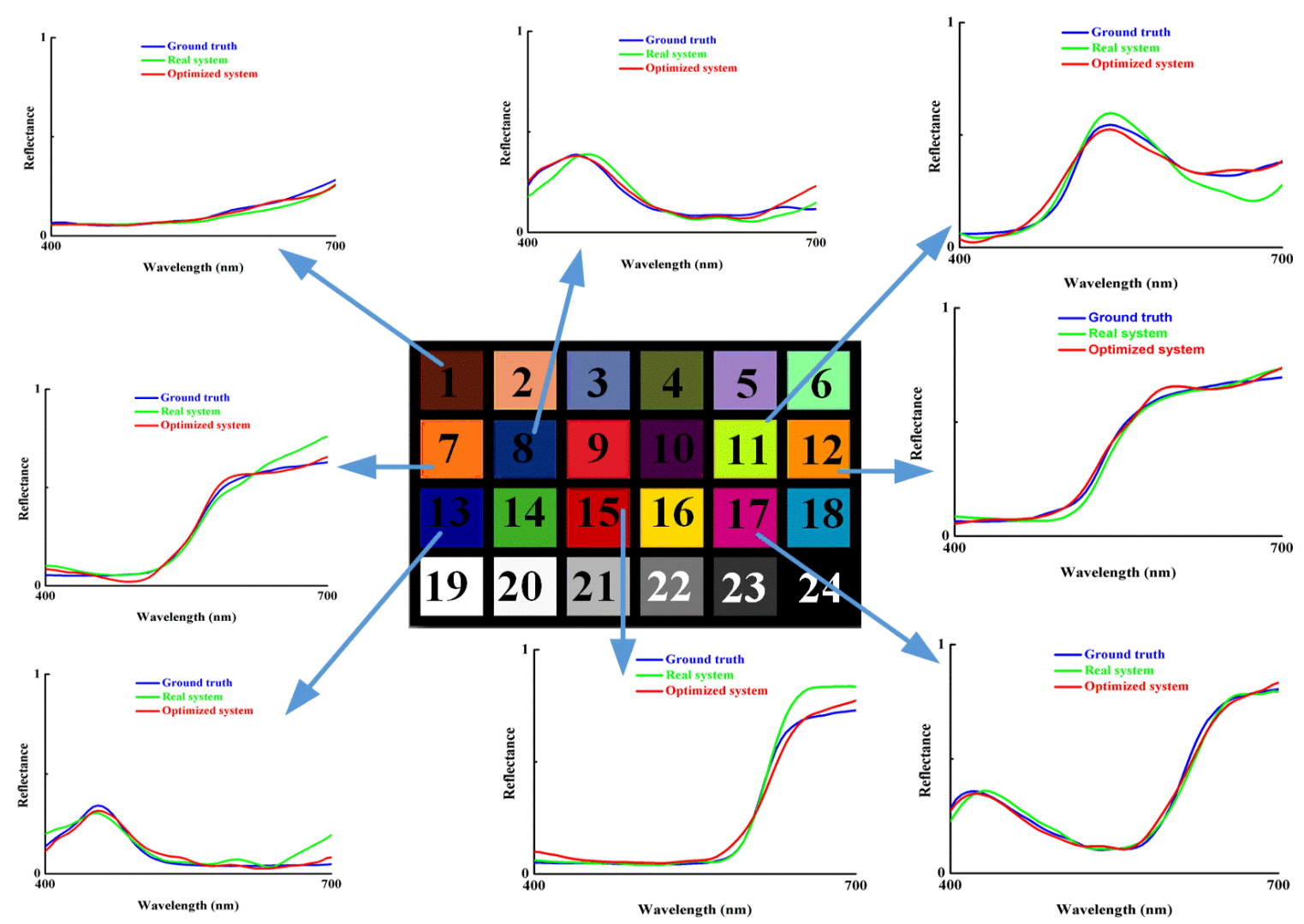

Fig. 8. A visual illustration of the improved estimation accuracy of optimized versus real system functions for a select number of Macbeth ColorChecker samples.

In addition, the mean RMSE, GFC, $\Delta u^{\prime} v^{\prime}$ and $\Delta Y$ values for real and optimized system functions are shown in Fig. 9 for all 24 reflectance samples of the Macbeth ColorChecker set. From Fig. 9(a) it is clear that, overall, the RMSE is rather stable across samples. Note that on average the RMSE is about 0.035 , with the largest RMSE value being less than 0.16 , indicating that optimized system function are capable of recovering sample spectral reflectance with good to reasonable accuracy. Importantly, optimized system functions lead, for the vast majority of samples, to RMSE values that are lower or equal to those obtained using a real system. From Fig. 9(b) it is clear that all GFC values are larger than 0.95, with the exception of one, indicating high curve similarity of the estimated reflectance with the ground truth. From Fig. 9(c) it can be seen that the CIE $1976 \Delta u^{\prime} v^{\prime}$ values, obtained using an 
optimized system, are typically less than 0.01 , with a few exceptions, while the $\Delta Y$ tend to be less than 0.05. Importantly, the colorimetric accuracy of the optimized system is also substantially better than the real system, as is also confirmed from the average values found in Fig. 7.
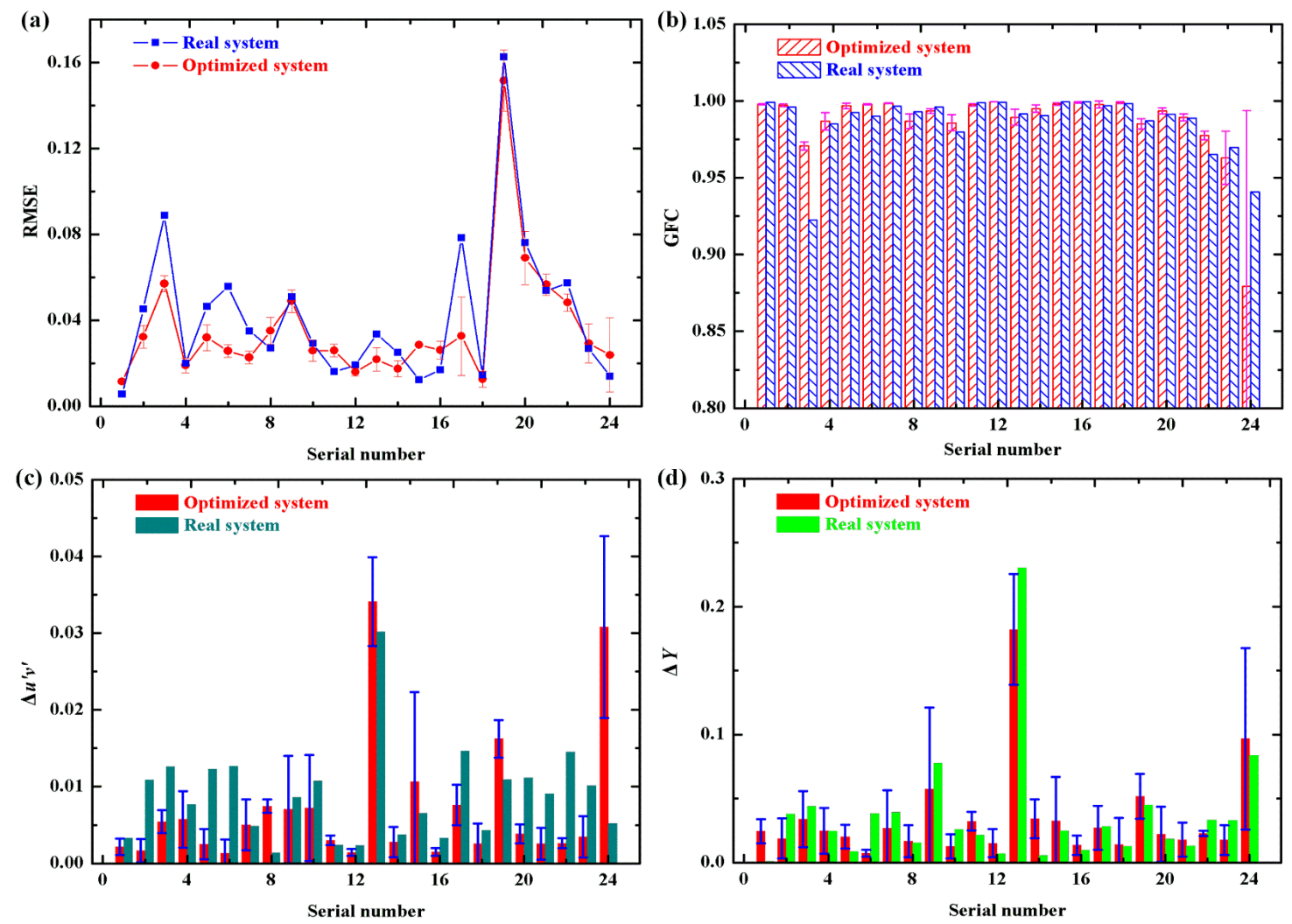

Fig. 9. Detailed estimation accuracy for the real and simulated systems for all 24 reflectance samples of the Macbeth ColorChecker data set in terms of for a) RMSE values; b) GFC; c) $\Delta u^{\prime} v^{\prime} ;$ d) $\Delta Y$.

It should be noted that, although multispectral cameras with optimized camera sensitivities can also perform well in obtaining spectral reflectances (Hardeberg 2004; Shimano 2006; Wang X et al. 2014), such multispectral cameras are expensive compared to a dual system with a cheap projector and a camera. 


\subsection{Robustness of optimal system functions under small parameter shifts}

For this analysis, one set of optimal system functions (see Fig. 10), obtained during previous analyses, was selected and its parameters (peak wavelength and FWHM) were shifted over $\pm 4 \mathrm{~nm}$. Note that FWHM of the camera sensitivities are approximate to the mean of the forward slope width and rear slope width parameters of the rounded triangle functions. Although the optimal projector spectra are different from commercially available projectors (shown in Fig. 1(a)), in this work we are exploring an optimized system from a theoretical point of view, i.e. what can be achieved given possible future developments. For example, not using a projector setup, but the RGB Philips hue LED lamp would result in quite broad peak in the $\mathrm{G}$ channel. Similar developments might also happen in projector design.
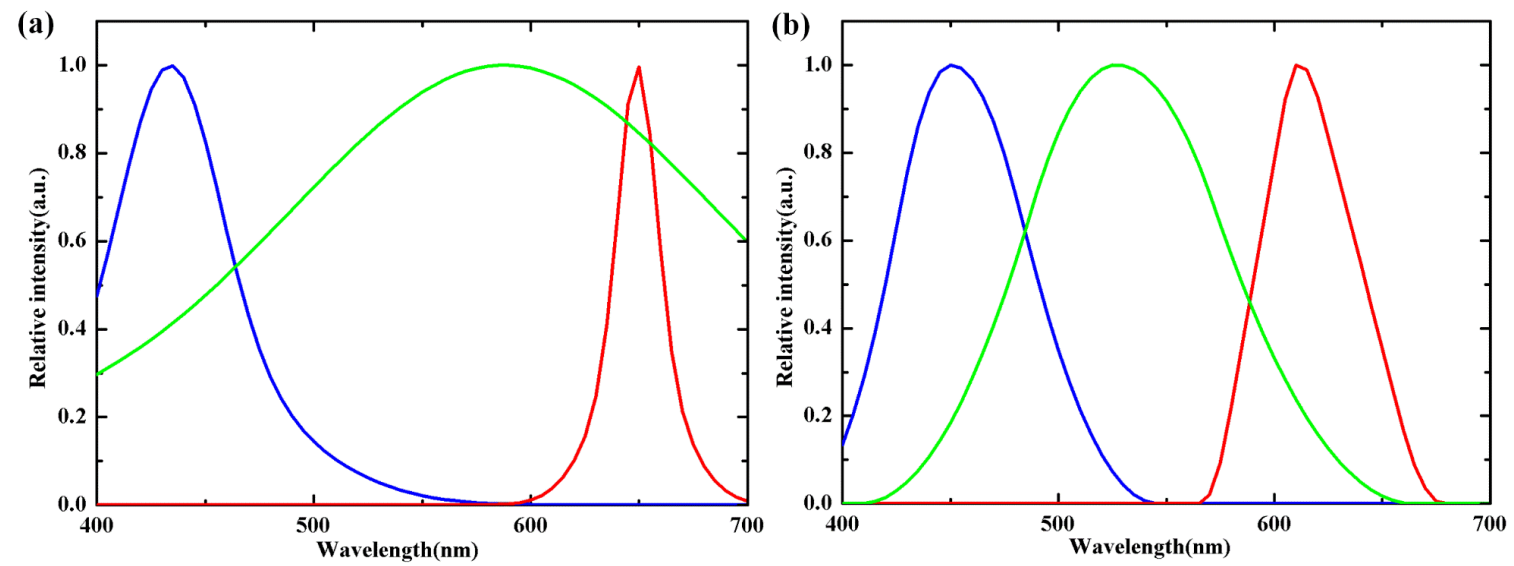

Fig. 10. One set of optimal projector spectra (a) and camera responsivity (b).

The mean RMSE difference between the shifted and unshifted systems for the Macbeth data set are shown in Fig. 11. From the figure, it is clear that the mean RMSE increases by less than 0.028 when shifting over a $\pm 4 \mathrm{~nm}$ range, indicating that the optimal system is quite stable for small parameter shifts with working temperature increases and passing time. It can also be observed that the most sensitive system function parameters are the red and green camera peak wavelengths and FWHM. Note that for some 
parameters (blue projector peak wavelength and blue projector FWHM), the difference in the mean RMSE between the shifted and unshifted systems is negative for small parameter shifts, because the unshifted system is not necessarily optimal in terms of mean RMSE (alone) when optimizing using the fitness function in Eq. 3.

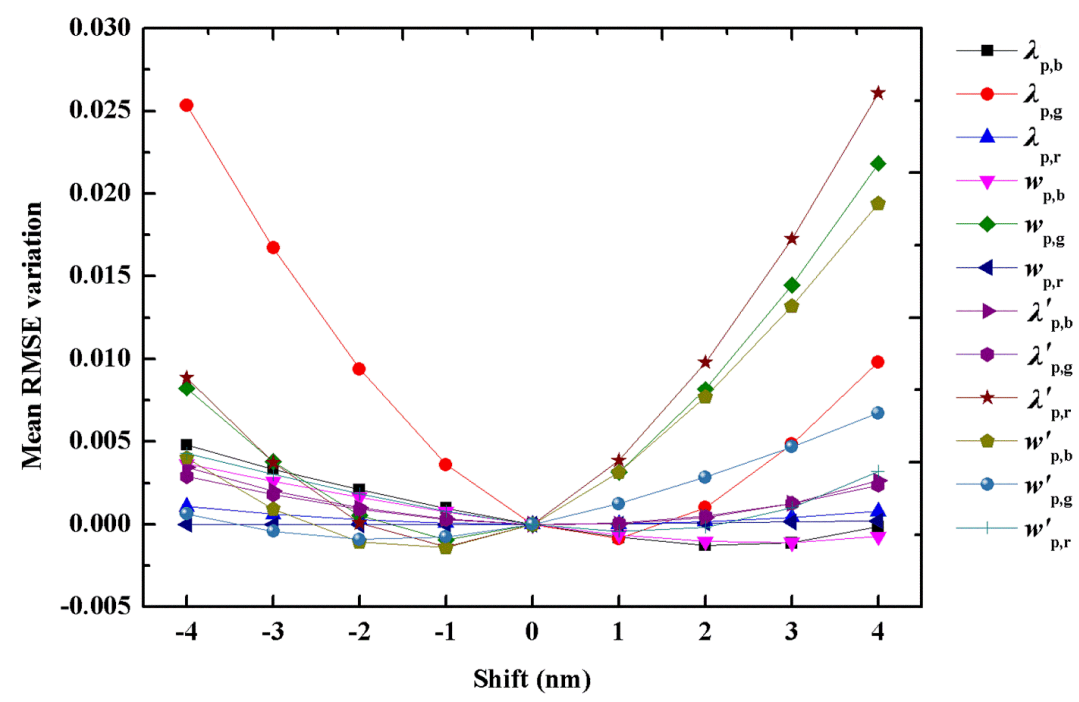

Fig. 11. Mean RMSE differences for the Macbeth data set with parameter shifts $\left(\lambda_{\mathrm{p}, \mathrm{b}}, \lambda_{\mathrm{p}, \mathrm{g}}\right.$, $\lambda_{\mathrm{p}, \mathrm{r}}$ refer to peak wavelength of blue, green, and red light source of projector; $w_{\mathrm{b}}, w_{\mathrm{g}}, w_{\mathrm{r}}$ refer to FWHM of blue, green, and red light source of projector, respectively. $\lambda_{\mathrm{p}, \mathrm{b}}^{\prime}, \lambda_{\mathrm{p}, \mathrm{g}}^{\prime}$, $\lambda_{\mathrm{p}, \mathrm{r}}^{\prime}$ refer to peak wavelength of blue, green, and red channel of camera; $w_{\mathrm{b}}^{\prime}, w_{\mathrm{g}}^{\prime}, w_{\mathrm{r}}^{\prime}$ refer to FWHM of blue, green, and red channel of camera, respectively).

Further, robustness has also been tested by systematically shifting a combination of the most two sensitive system function parameters (red and green camera peak wavelengths) over a $\pm 4 \mathrm{~nm}$ range in steps of $1 \mathrm{~nm}$ and calculating the mean RMSE values for the Macbeth data set using the shifted system functions. The mean RMSE difference between the shifted and unshifted systems for the Macbeth data set are shown in Fig. 12. It is found that when the peak wavelength deviation of red light source and the blue camera are resp. $-4 \mathrm{~nm}$ and $4 \mathrm{~nm}$, that the mean RMSE difference is largest with a value of 0.048 , which is less than the sum of the mean RMSE differences obtained when only the red or only the green camera system functions are shifted by $4 \mathrm{~nm}$. Note that from 
Fig. 11 and Fig. 12, it can also be seen that the situation is the same for other peak wavelength deviations. Therefore, it can be concluded that the mean RMSE effect caused by different system functions is not additive.

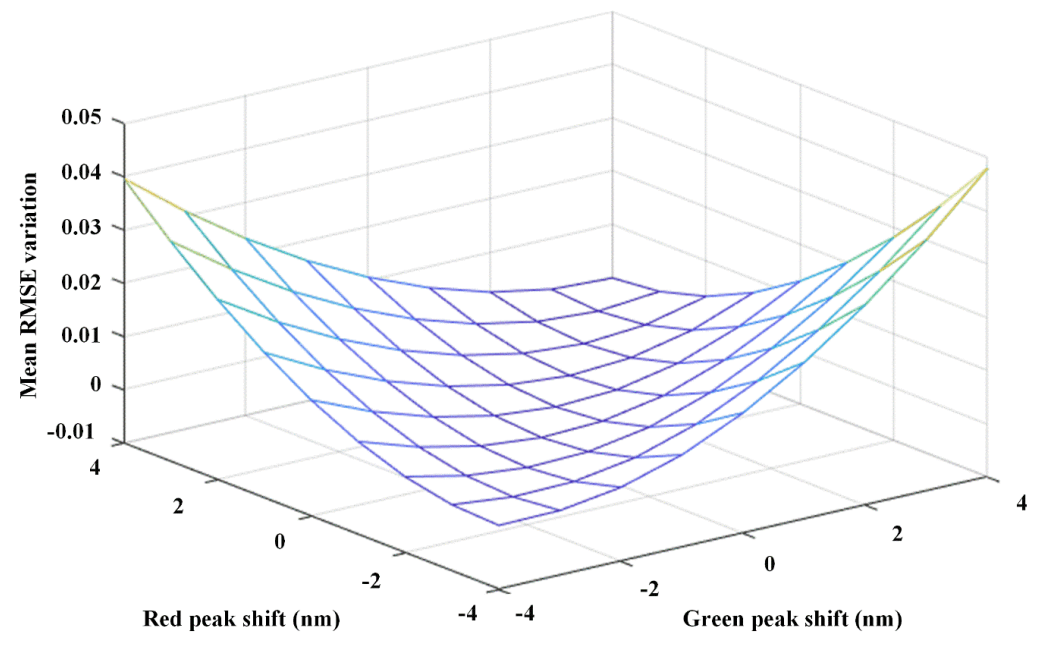

Fig. 12. Mean RMSE differences for the Macbeth data set for peak wavelength shifts of the red light source, and the blue camera responsivities.

\subsection{Impact of the sample set database used for neural network training on optimal system performance.}

Optimal system performance was investigated for training and system function parameter optimization using three different training sample sets, all limited to a common cylindrical gamut as described in section 2.6. The performance of each optimized system, assessed as the fitness value $F$ (see Eq. 3) between ground-truth and estimate spectral reflectance and subject to the $\mathrm{SMI} \geq 95$ constraint, for several validation sample sets, are shown in Fig. 13.

From Fig. 13 it is clear that the performance tends to be the highest when the training set comes from the same database as the validation set. For example, training with a Munsell based set shows the highest performance (lowest mean RMSE, $\Delta u^{\prime} v^{\prime}$ and $\Delta Y$ values) when the validation set is also Munsell based, but can be substantially higher for some other validation sets. The same can be found for the Leeds samples (albeit with 
a slight better performance in terms of mean RMSE). These results for the cylindrically limited validation sets suggest that the IES set is more stable and better at representing the overall spectral characteristics of other samples sets, which was one of the design intents (i.e. spectral uniformity) of the set.
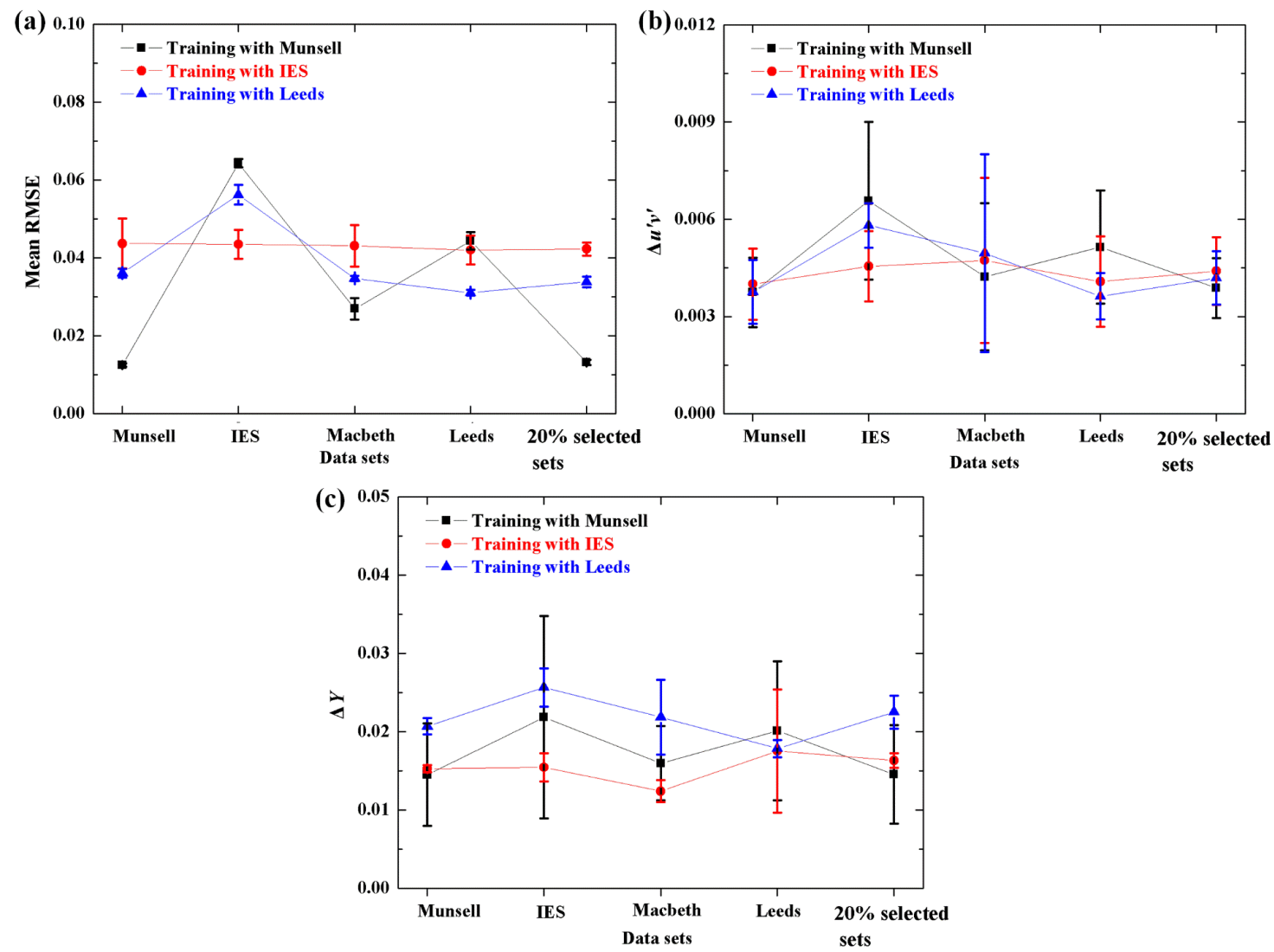

Fig. 13. Reflectance estimation performance in terms of a) mean RMSE, b) $\Delta u^{\prime} v^{\prime}$ and c) $\Delta Y$ values for various validation sample sets for systems optimized and trained using the Munsell, IES and Leeds sample sets limited to a common cylindrical volume.

\subsection{A comparison of accuracy and running speed of the proposed method with}

\section{Liang's method}

\subsubsection{Accuracy}

The proposed method was compared to Liang's original method (Liang et al. 2019) and an extended version with optimized system functions. As described in section 2.7, training and optimization sets were randomly drawn (without repetition) from and with a 
size of $60 \%$ and a size of $20 \%$ of the selected IES subset which was limited to the minimal cylindrical volume. The performance of the methods, evaluated as the mean RMSE between the ground-truth and the estimations for the full (i.e. 100\%) IES, full Leeds, full Macbeth and full Munsell sets, but limited to the minimal cylindrical volumes as described in section 2.6, are shown in Fig. 14 (a). The color differences $\left(\Delta u^{\prime} v^{\prime}\right)$ between the ground-truth and the estimations under illuminant $\mathrm{E}$ and a group of 318 lamp spectra published in the IES-30-15 Excel calculator (David et al. 2015) are shown in Fig.14 (b) and Fig.14 (c), respectively.

It can be seen from Fig. 14(a) that our proposed method has slightly higher RMSE values but lower color difference $\left(\Delta u^{\prime} v^{\prime}\right)$ values after optimization of the system function than Liang's method. Note that Fig. 14(c) shows that the proposed method, on average, results in substantially better color predictions under lamp spectra that are different from the 'training' lamp spectrum (illuminant E). The proposed method is therefore more robust with regard to uncertainties in the actual shape of the illuminating lamp spectrum or under complete changes in lamp spectrum all together. The results also suggest, that Liang's method might be overfitting some parts of the spectral reflectance functions that are not that crucial or influential for color vision and underfitting other parts. However, the proposed method strikes a good balance between RMSE and color difference (comparable to the average just-noticeable-difference or JND; note that $1 \mathrm{JND} \approx$ $0.0033 u^{\prime} v^{\prime}$ units in white region (Ohno Y and Blattner 2014), but overall average JND $\approx$ $0.0047 u v^{\prime}$ units). In addition, the estimation error by the proposed method can be further decreased when noise is reduced as imaging technology develops. 

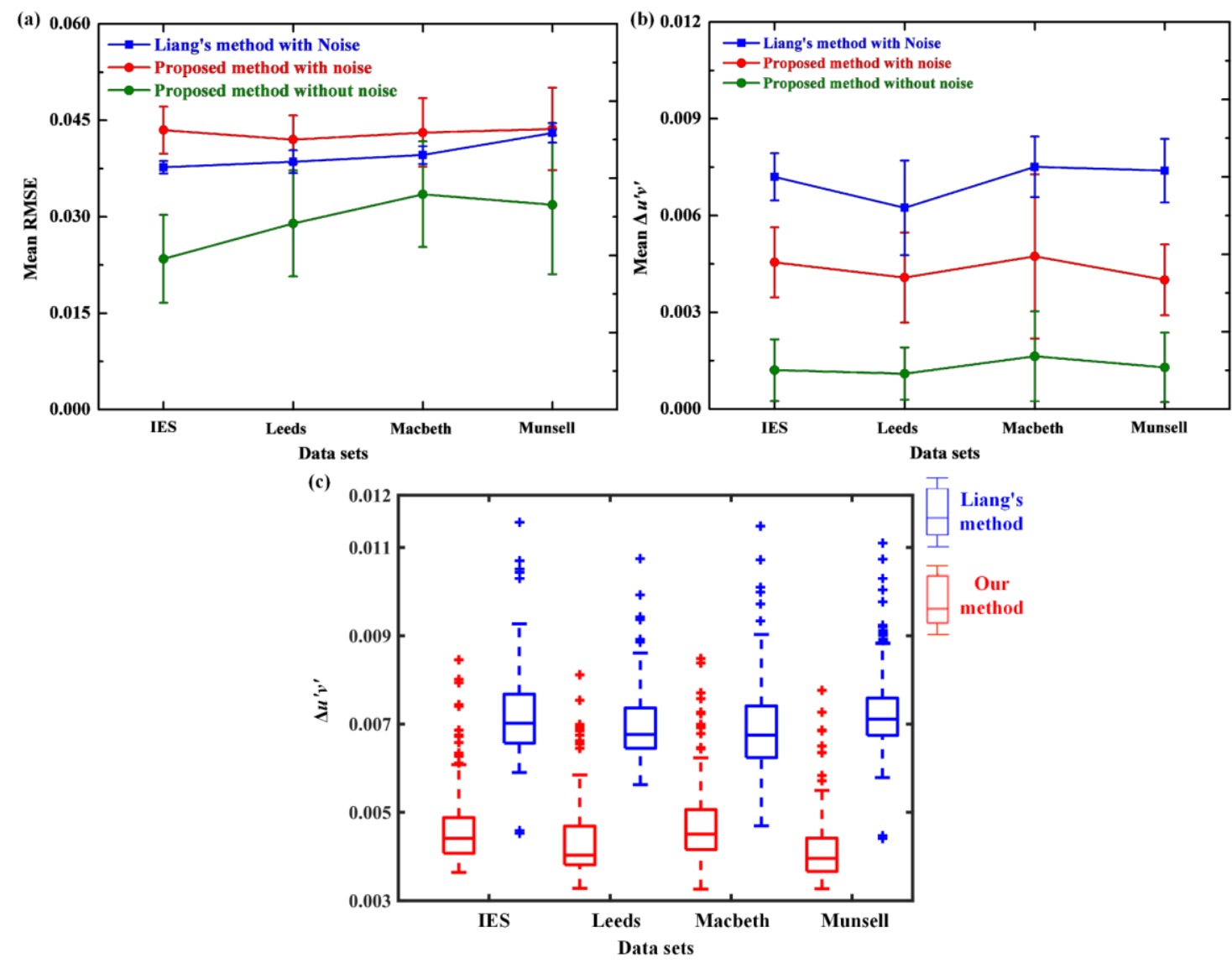

Fig. 14. Accuracy comparison between the proposed method and Liang's method with optimized system functions. a. Mean RMSE values, b. Mean $u^{\prime} v^{\prime}$ color differences under illumination by an equi-energy spectrum, c. $u^{\prime} v^{\prime}$ color differences distribution under illumination by a group of 318 lamp spectra.

\subsubsection{Speed}

Results for the running times of the proposed method and Liang's original method are shown in Fig. 15. As can be seen, running times for the proposed method are much lower than the ones for Liang's method, because neural network training and system optimization can all be done in advance, after which reflectance estimation for all scene pixel can be done virtually simultaneously. On the other hand, Liang's method requires color distance calculation for each unique pixel in an image. For images, with an average color diversity of $18.3 \%$ (Quattoni and Torralba 2009) and resolution of 512 pixels by 512 pixels, the running time is approximately 1924 seconds, while it is barely 0.35 second 
for the proposed method. At typical image resolution (1024 pixels by 1024 pixels) (Specim 2018) of hyperspectral cameras, Liang's method is impractical both from a running time (6700 seconds) and memory requirement point of view. However, with a running time less than 2 seconds, the proposed method is.

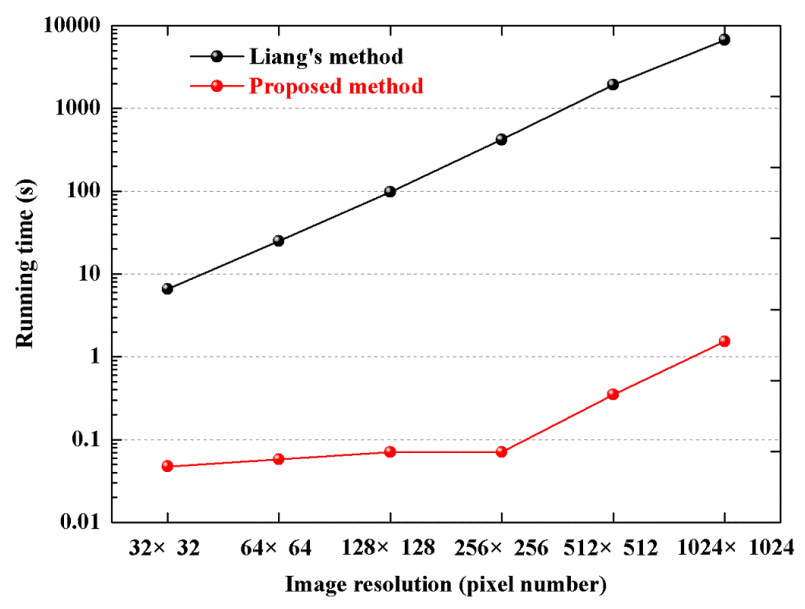

Fig. 15. Running time of the proposed method and Liang's as a function of the image resolution.

\section{Conclusions}

The spectral reflectance estimation accuracy was investigated for real and simulated theoretical dual imaging systems composed of a 3-channel projector as light source and a 3-channel camera. The accuracy of such dual systems was shown to vary greatly depending on the system function (light source SPDs and camera responsivities) characteristics, such as peak wavelength and FWHM. Optimization of the latter in the simulated systems lead to a substantially improved accuracy compared to what can be achieved using commercially available projector and camera combination. Consequently, light source spectra and camera responsivity play a very important role in the performance of reflectance estimation accuracy.

A test of the robustness of the optimized system under small individual variations ( $\pm 4 \mathrm{~nm}$ shifts) of the peak wavelength and FWHM of the system functions showed that 
the mean RMSE difference between the shifted and unshifted system was less than 0.028 . The largest mean RMSE occurred for shifts of the peak wavelengths of red light source and the blue camera responsivity curves. In addition, the mean RMSE difference between the shifted and unshifted system remained less than 0.048 under small combinational variations ( $\pm 4 \mathrm{~nm}$ shifts) of the peak wavelengths of green and red camera responsivities. Optimal parameters of projector spectra and camera responsivities are therefore sufficiently robust and stable for the small parameter shifts associated with working temperature increases and passing time.

Results of training and system function parameter optimization using three different training sample sets (Munsell set, IES set, Leeds set) showed that training with IES set had good and stable performance for other sample sets (Munsell, Leeds and Macbeth ColorChecker) as well, suggesting the IES set is best at representing the overall spectral characteristics of other samples sets. Training with the spectrally uniform IES spectral reflectance set (David et al. 2015; Smet Kevin A. G. et al. 2016; IES 2018) is therefore advised for general purpose, high-accuracy reflectance estimation systems.

Finally, a comparison of accuracy and running time with a state-of-the-art method published in literature (Liang et al. 2019), shows that the proposed method has a higher color prediction accuracy, is more robust under changes or uncertainties in illuminant spectrum and has a significantly shorter running time. It's concluded that the neural net combined with optimized system functions and trained with the IES TM30 spectral reflectance set provides a good technical solution to estimate spectral reflectance for real, general purpose scenes and applications. 


\section{Acknowledgments}

The work was carried out at ESAT/Light \& Lighting Laboratory, KU Leuven, Ghent, Belgium.

J. Zhang thanks Prof. Peter Hanselaer and colleagues at the Lab for all the help.

\section{Funding}

This work was supported by National Natural Science Foundation of China (NSFC) (61604135), China Scholarship Council (201706415021), Fundamental Research Funds for the Central Universities (CUGL180404), and Internal funds KU Leuven.

\section{Disclosure statement}

The authors have no financial interests to declare.

\section{References}

AEON Imaging L. 2015. DLM confocal microscope. [accessed 2015 Dec 15]. https://www.aeonimaging.com/dlm-confocal-microscope-technology/.

Ali MH, Lyon P, Meerleer PD. 2014. Night vision goggle stimulation using LCoS and DLP projection technology, which is better? Proc SPIE 9086, Display Technologies and Applications for Defense, Security, and Avionics VIII; and Head- and Helmet-Mounted Displays XIX.

Arad B, Ben-Shahar O. 2016. Sparse recovery of hyperspectral signal from natural RGB images. European Conference on Computer Vision.

Burggraaff O, Schmidt N, Zamorano J, Pauly K, Pascual S, Tapia C, Spyrakos E, Snik F. 2019. Standardized spectral and radiometric calibration of consumer cameras. Opt Express. 27(14):19075-19101.

Cao B, Liao NF, Cheng HB. 2017. Spectral reflectance reconstruction from RGB images based on weighting smaller color difference group. Color Research and Application. 42(3):327-332.

Chi C, Yoo H, Ben-Ezra M. 2010. Multi-spectral imaging by optimized wide band illumination. International Journal of Computer Vision. 86(2-3):140-151.

mCo C-s. 1997. The Basis of Color Reproduction Engineering.

Coburn CA, Smith AM, Logie GS, Kennedy P. 2018. Radiometric and spectral comparison of inexpensive camera systems used for remote sensing. International Journal of Remote Sensing. 39(15-16):4869-4890.

Dana KJ. 2016. Capturing computational appearance: more than meets the eye. IEEE Signal Processing Magazine. 33(5):70-80.

David A, Fini PT, Houser KW, Ohno Y, Royer MP, Smet KAG, Minchen, Wei, Whitehead L. 2015. Development of the IES method for evaluating the color rendition of light sources. Opt Express. 23(12):15888-11590.

de Miguel AS, Kyba CC, Aubé M, Zamorano J, Cardiel N, Tapia C, Bennie J, Gaston KJ. 2019. Colour remote sensing of the impact of artificial light at night (I): The potential of the International Space Station and other DSLR-based platforms. Remote sensing of environment. 224:92-103.

Deb K. 2000. An efficient constraint handling method for genetic algorithms. Computer Methods in Applied Mechanics and Engineering. 186(2-4):311-338.

Deeb R, Van de Weijer J, Muselet D, Hebert M, Tremeau A. 2019. Deep spectral reflectance and illuminant estimation from self-interreflections. J Opt Soc Am A. 36(1):105-114.

Delica S, Blanca CM. 2007. Wide-field depth-sectioning fluorescence microscopy using projector-generated patterned illumination. Applied Optics. 46(29):7237-7243.

Dixon EL, Shapiro AG. 2017. Spatial filtering, color constancy, and the color-changing dress. J Vision. 17(3):7,1-20. 
Durmus D, Abdalla D, Duis A, Davis W. 2020. Spectral Optimization to Minimize Light Absorbed by Artwork. Leukos. 16(1):45-54.

Durmus D, Davis W. 2015. Optimising light source spectrum for object reflectance. Opt Express. 23(11):A456-A464.

Durmus D, Davis W. 2018. Appearance of Achromatic Colors Under Optimized Light Source Spectrum. Ieee Photonics Journal. 10(6).

Familiarization with Properties of Light Sources. 2010. [accessed 2010 Aug 10]. https://colorscience.wordpress.com/2010/08/07/2-familiarization-with-properties-of-lightsources/.

Filip J, Vavra R, Havlicek M, Krupicka M. 2017. Predicting visual perception of material structure in virtual environments. Computer Graphics Forum. 36(1):89-100.

Foi A, Trimeche M, Katkovnik V, Egiazarian K. 2008. Practical poissonian-gaussian noise modeling and fitting for single-image raw-data. IEEE Transactions on Image Processing. 17(10):1737-1754.

Fu X, Liao Y, Zeng D, Huang Y, Zhang XP, Ding X. 2015. A probabilistic method for image enhancement with simultaneous illumination and reflectance estimation. IEEE Transactions on Image Processing. 24(12):4965-4977.

Fu Y, Zhang T, Zheng Y, Zhang D, Huang H. 2018. Joint camera spectral sensitivity selection and hyperspectral image recovery. European Conference on Computer Vision.

Georgoulis S, Rematas K, Ritschel T, Gavves E, Fritz M, Gool LV, Tuytelaars T. 2017. Reflectance and natural Illumination from single-material specular objects using deep learning. IEEE Transactions on Pattern Analysis and Machine Intelligence. 40(8):1932-1947.

Grusche S, Theilmann F. 2015. An RGB approach to extraordinary spectra. European Journal of Physics. 36(5):055018.

Han S, Sato I, Okabe T, Sato Y. 2013. Fast spectral reflectance recovery using DLP projector. International Journal of Computer Vision. 110(2):172-184.

Hardeberg JY. 2004. Filter selection for multispectral color image acquisition. Journal of Imaging Science and Technology. 48(2):105-110.

Hasinoff SW. 2014. Photon, poisson noise. In: Ikeuchi K, editor. Computer Vision: A Reference Guide. Boston, MA: Springer US; p. 608-610.

Healey G. 1991. Estimating spectral reflectance using highlights. Image and Vision Computing. 9(5):333-337.

Healey GE, Kondepudy R. 1994. Radiometric CCD camera calibration and noise estimation. IEEE Transactions on Pattern Analysis and Machine Intelligence. 16(3):267-276.

Heikkinen V. 2018. Spectral reflectance estimation using gaussian processes and combination kernels. IEEE Transactions on Image Processing. 27(7):3358-3373.

Heikkinen V, Cámara C, Hirvonen T, Penttinen N. 2016. Spectral imaging using consumer-level devices and kernel-based regression. J Opt Soc Am A. 33(6):1095-1110.

Heikkinen V, Lenz R, Jetsu T, Parkkinen J, Hauta-Kasari M, Jääskeläinen T. 2008. Evaluation and unification of some methods for estimating reflectance spectra from RGB images. J Opt Soc Am A. 25(10):2444-2458.

IES. 2018. TM-30-18: IES method for evaluating light source color rendition. The Illuminating Engineering Society of North America.

Jiang J, Gu J. 2012. Recovering spectral reflectance under commonly available lighting conditions. IEEE Computer Society Conference on Computer Vision and Pattern Recognition Workshops.

Jiang J, Liu D, Gu J, Süsstrunk S. 2013. What is the space of spectral sensitivity functions for digital color cameras? IEEE Workshop on Applications of Computer Vision (WACV).

Khan R, Joost VDW, Karatzas D, Muselet D. 2013. Towards multispectral data acquisition with hand-held devices. IEEE International Conference on Image Processing.

l'Eclairage CId. 2005. Colormetry. Vienna, Austria. Technical Report, CIE 15 No.

Lam A, Subpa-Asa A, Sato I, Okabe T, Sato Y. 2013. Spectral imaging using basis lights.

Li C, Ronnier Luo M, Li C, Cui G. 2012. The CRI-CAM02UCS colour rendering index. Color Research \& Application. 37(3):160-167. 
Li S-X. 2018. Filter selection for optimizing the spectral sensitivity of broadband multispectral cameras based on maximum linear independence. Sensors. 18(5):1455.

Liang J, Xiao K, Pointer MR, Wan X, Li C. 2019. Spectra estimation from raw camera responses based on adaptive local-weighted linear regression. Opt Express. 27(4):5165-5180.

Liu C, Szeliski R, Kang SB, Zitnick CL, Freeman WT. 2008. Automatic estimation and removal of noise from a single image. IEEE Transactions on Pattern Analysis and Machine Intelligence. 30(2):299-314.

Liu Z, Liu Q, Gao GA, Li C. 2017. Optimized spectral reconstruction based on adaptive training set selection [Article]. Opt Express. 25(11):12435-12445. English.

Liu Z, Wan XX, Huang XG, Liu Q, Li C. 2013. [The study on spectral reflectance reconstruction based on wideband multi-spectral acquisition system]. Spectrosc Spect Anal. 33(4):1076.

Maloney LT, Wandell BA. 1986. Color constancy - A method for recovering surface spectral reflectance. J Opt Soc Am A-Opt Image Sci Vis. 3(1):29-33.

Nahavandi AM, Tehran MA. 2017. A new manufacturable filter design approach for spectral reflectance estimation. Color Research and Application. 42(3):316-326.

Ng D-Y, Allebach JP. 2006. A subspace matching color filter design methodology for a multispectral imaging system. IEEE Transactions on Image Processing. 15(9):2631-2643.

Nguyen RMH, Prasad DK, Brown MS. 2014. Training-based Spectral Reconstruction from a Single RGB Image. 13th European Conference on Computer Vision , 6-12 Sept; Cham, Switzerland.

Nie S, Gu L, Zheng Y, Lam A, Ono N, Sato I. 2018. Deeply learned filter response functions for hyperspectral reconstruction. IEEE Conference on Computer Vision and Pattern Recognition.

Oh SW, Brown MS, Pollefeys M, Kim SJ, Ieee. 2016. Do it yourself hyperspectral imaging with everyday digital cameras. IEEE Conference on Computer Vision and Pattern Recognition; New York.

Ohno Y. 2005. Spectral design considerations for white LED color rendering. Optical Engineering. 44(11):111302.

Ohno Y, Blattner P. 2014. Chromaticity difference specification for light sources. International Commission on Illumination, Tech Rep CIE TN. 1:2014.

Oxholm G, Nishino K. 2016. Shape and Reflectance Estimation in the Wild. IEEE Transactions on Pattern Analysis and Machine Intelligence. 38(2):376-389.

Packer O, Diller LC, Verweij J, Lee BB, Pokorny J, Williams DR, Dacey DM, Brainard DH. 2001. Characterization and use of a digital light projector for vision research. Vision Research. 41(4):427-439.

Park JI, Lee MH, Grossberg MD, Nayar SK, Ieee. 2007. Multispectral imaging using multiplexed illumination. IEEE 11th International Conference on Computer Vision. p. 1-8.

Perlova T. 2014. Measured LightCrafter LED spectra. [accessed 2014 Feb 21]. https://e2e.ti.com/support/dlp_mems_micro-electromechanical_systems/advanced_light_control/f/850/t/323184.

Prucnal PR, Saleh BEA. 1981. Transformation of image-signal-dependent noise into imagesignal-independent noise. Opt Lett. 6(7):316-318.

Quattoni A, Torralba A. 2009. Recognizing indoor scenes. IEEE Conference on Computer Vision and Pattern Recognition.

Raypah ME, Devarajan M, Sulaiman F. 2017. Modeling spectra of low-power SMD LEDs as a function of ambient temperature. IEEE Trans Electron Devices. 64(3):1180-1186.

Samson EC, Blanca CM. 2007. Dynamic contrast enhancement in widefield microscopy using projector-generated illumination patterns. New Journal of Physics. 9:363.

Schubert EF, Kim JK. 2005. Solid-state light sources getting smart. Science. 308(5726):12741278.

Sharma G, Trussell HJ. 1997. Figures of merit for color scanners. IEEE Transactions on Image Processing. 6(7):990-1001.

Shen J, Chang S, Wang H, Zheng Z. 2019. Optimising the illumination spectrum for enhancing tissue visualisation. Lighting Research \& Technology. 51(1):99-110.

Shimano N. 2006. Optimization of spectral sensitivities with Gaussian distribution functions for a color image acquisition device in the presence of noise. Optical Engineering. 45(1):013201. 
Shrestha R, Mansouri A, Hardeberg JY. 2011. Multispectral imaging using a stereo camera: concept, design and assessment. EURASIP Journal on Advances in Signal Processing. 2011(1):57.

Smet KAG, David A, Whitehead L. 2016. Why color space uniformity and sample set spectral uniformity are essential for color rendering measures. LEUKOS. 12(1-2):39-50.

Smet KAG, Schanda J, Whitehead L, Luo RM. 2013. CRI2012: A proposal for updating the CIE colour rendering index [Article]. Lighting Research \& Technology. 45(6):689-709. English.

Specim SIL. 2018. Specim FX Series. [accessed 2019]. http://www.specim.fi/fx/\#1478603793717-9356cb24-923f.

Stirman JN, Crane MM, Husson SJ, Gottschalk A, Lu H. 2012. A multispectral optical illumination system with precise spatiotemporal control for the manipulation of optogenetic reagents. Nature Protocols. 7(2):207-220.

Technologies A. LED projectors versus UHP projectors. [accessed 2014]. http://www.aaxatech.com/articles/LEDversusUHP.htm.

Tominaga S. 1996. Multichannel vision system for estimating surface and illumination functions. J Opt Soc Am A-Opt Image Sci Vis. 13(11):2163-2173.

Tominaga S, Okajima R. 2000. Object recognition by multi-spectral imaging with a liquid crystal filter. 15th International Conference on Pattern Recognition.

mTrussell J. 2011. System and method for estimating projector primary spectra using rgb measurement. Google Patents.

Tu GJ, Karstoft H, Pedersen LJ, Jørgensen E. 2015. Illumination and reflectance estimation with its application in foreground detection. Sensors. 15(9):21407-21426.

Urban P, Rosen MR, Berns RS. 2009. Spectral image reconstruction using an edge preserving spatio-spectral Wiener estimation. J Opt Soc Am A. 26(8):1865-1875.

Vora PL, Trussell HJ. 1993. Measure of goodness of a set of color-scanning filters. J Opt Soc Am A. 10(7):1499-1508.

Wang H, Cuijpers RH, Vogels I, Luo MR, Heynderickx I, Zheng Z. 2018. Optimising the illumination spectrum for tissue texture visibility. Lighting Research \& Technology. 50(5):757-771.

Wang X, Thomas J-B, Hardeberg JY, Gouton P. 2014. Multispectral imaging: narrow or wide band filters? JAIC-Journal of the International Colour Association. 12.

Xiao K, Zhu Y, Li C, Connah D, Yates JM, Wuerger S. 2016. Improved method for skin reflectance reconstruction from camera images. Opt Express. 24(13):14934-14950.

Xie B, Hu R, Luo XB. 2016. Quantum dots-converted light-emitting diodes packaging for lighting and display: status and perspectives. Journal of Electronic Packaging. 138(2):020803.

Xie B, Zhang J, Chen W, Hao J, Cheng Y, Hu R, Wu D, Wang K, Luo X. 2017. Realization of wide circadian variability by quantum dots-luminescent mesoporous silica-based white lightemitting diodes. Nanotechnology. 28(42):425204.

Spectrally-based single image relighting. 2010; Berlin, Heidelberg. Springer Berlin Heidelberg.

Xu G-Q, Zhang J-H, Cao G-Y, Xing M-S, Li D-S, Yu J-J. 2017. Solar spectrum matching using monochromatic LEDs. Lighting Research \& Technology. 49(4):497-507.

Zhang J, Hu R, Xie B, Yu X, Luo X, Yu Z, Zhang L, Wang H, Jin X. 2017. Energy-saving light source spectrum optimization by considering object's reflectance. IEEE Photonics Journal. 9(2):8200311.

Zhang J, Hu R, Yu X, Xie B, Luo X. 2017. Spectral optimization based simultaneously on colorrendering index and color quality scale for white LED illumination. Optics \& Laser Technology. 88:161-165.

Zhang J, Smet KAG, Meuret Y. 2019. Tuning color and saving energy with spatially variable laser illumination. Opt Express. 27(19):27136-27150.

Zhang JJ, Smet KAG, Meuret Y. 2019. Tuning color and saving energy with spatially variable laser illumination. Opt Express. 27(19):27136-27150.

Zhang JJ, Zhang T, Liu SS, Yuan SD, Jin YF, Yang S. 2013. Feasibility analysis of junction temperature measurement for GaN-based high-power white LEDs by the peak-shift method. Chinese Optics Letters. 11(9):091204. 
Zhang X, Wang Q, Li J, Zhou X, Yang Y, Xu H. 2017. Estimating spectral reflectance from camera responses based on CIE XYZ tristimulus values under multi-illuminants. Color Research and Application. 42(1):68-77.

Zhang $\mathrm{X}, \mathrm{Xu} \mathrm{H}$. 2008. Reconstructing spectral reflectance by dividing spectral space and extending the principal components in principal component analysis. J Opt Soc Am A. 25(2):371-378.

Estimating basis functions for spectral sensitivity of digital cameras. Meeting on Image Recognition and Understanding; 2009.

Zhao Y, Yun F, Feng L, Wang S, Li Y, Su X, Guo M, Ding W, Zhang Y. 2017. Improving peakwavelength method to measure junction temperature by dual-wavelength LEDs. IEEE Access. 5(99):11712-11716. 